\title{
On a Family of Quasimetric Spaces in Generalized Potential Theory
}

\author{
Per Åhag ${ }^{1} \cdot$ Rafał Czyż ${ }^{2}$
}

Received: 11 October 2021 / Accepted: 16 December 2021 / Published online: 29 January 2022

(c) The Author(s) 2022

\begin{abstract}
We construct a family of quasimetric spaces in generalized potential theory containing $m$-subharmonic functions with finite $(p, m)$-energy. These quasimetric spaces will be viewed both in $\mathbb{C}^{n}$ and in compact Kähler manifolds, and their convergence will be used to improve known stability results for the complex Hessian equations.
\end{abstract}

Keywords Caffarelli-Nirenberg-Spruck model · Cegrell class · Complex Hessian operator $\cdot m$-subharmonic function $\cdot$ Quasimetric space $\cdot$ Stability

Mathematics Subject Classification Primary 32U05 - 31C45; Secondary 31E05 . 46E36

\section{Introduction}

Although the abstract metric spaces introduced by Fréchet at the beginning of the last century are of the utmost importance, in some applications, they are too restrictive and need a more general model. To name a few examples: the Minkowski $p$-distance in

We raise our cups to Urban Cegrell, gone but not forgotten, gone but ever here. Until we meet again in Valhalla!

The second-named author was supported by the Priority Research Area SciMat under the program Excellence Initiative - Research University at the Jagiellonian University in Kraków.

$凶$ Per Åhag

Per.Ahag@math.umu.se

Rafał Czyż

Rafal.Czyz@im.uj.edu.pl

1 Department of Mathematics and Mathematical Statistics, Umeå University, 90187 Umeå, Sweden

2 Institute of Mathematics Faculty of Mathematics and Computer Science, Jagiellonian University, Łojasiewicza 6, 30-348 Kraków, Poland 
psychology $(p<1)$ [27,36], the Zolotarev distance in spaces of random variables [40], and the $d_{\epsilon}$-distance in machine learning [13]. The terminology has not yet stabilized within the many generalizations of metric spaces and therefore let us determine which one we will work with here. Let $X$ be a non-empty set, and let $d: X \times X \rightarrow[0, \infty)$ be a function that satisfies:

(1) $d(x, y)=0$ if, and only if, $x=y$;

(2) $d(x, y)=d(y, x)$, for all $x, y \in X$;

(3) there exists a constant $C \geq 1$ such that

$$
d(x, y) \leq C(d(x, z)+d(z, y))
$$

for all $x, y, z \in X$.

Following for example Heinonen [26], we shall call $d$ for a quasimetric, and the pair $(X, d)$ for a quasimetric space. Some writers call this instead for a nearmetric or inframetric. Next, let us define our specific $X$ and then construct $d$.

Let $n \geq 2$ and $1 \leq m \leq n$. We say that a $\mathcal{C}^{2}$-function $u$ defined in a bounded domain in $\mathbb{C}^{n}$ is $m$-subharmonic if the elementary symmetric functions are positive $\sigma_{l}(\lambda(u)) \geq 0$ for $l=1, \ldots, m$, where $\lambda(u)=\left(\lambda_{1}, \ldots, \lambda_{n}\right)$ are eigenvalues of the complex Hessian matrix $D_{\mathbb{C}}^{2} u=\left[\frac{\partial^{2} u}{\partial z_{j} \partial \bar{z}_{k}}\right]$. The complex $m$-Hessian operator on a $\mathcal{C}^{2}$-function $u$ is then defined by

$$
\mathrm{H}_{m}(u)=c(n, m) \sigma_{m}\left(\lambda\left(D_{\mathbb{C}}^{2} u\right)\right)
$$

for some constant $c(n, m)$ depending only on $n$ and $m$.

This construction yields that the 1-Hessian operator is the Laplace operator defined on 1-subharmonic functions that are just the subharmonic functions, while the complex $n$-Hessian operator is the complex Monge-Ampère operator defined on $n$-subharmonic functions that are the plurisubharmonic functions. Historically this model goes back to Caffarelli et al. [14] in 1985, where they did a similar construction for the real Hessian matrix. Vinacua, a student of Nirenberg, was one of those who adapted the idea of the Hessian operator to the complex setting ( $[38,39])$ that we shall use here. Later in 2005, Błocki [12] introduced pluripotential methods to the theory of complex Hessian operators, and there he, among other things, generalized the complex Hessian operator to non-smooth $m$-subharmonic functions. For $p>0$, set

$$
e_{p, m}(u)=\int_{\Omega}(-u)^{p} \mathrm{H}_{m}(u)
$$

and we shall call $e_{p, m}(u)$ for the $(p, m)$-energy of the function $u$. Let $\mathcal{E}_{p, m}(\Omega)$ be the class of $m$-subharmonic functions that, in a general sense, vanish on the boundary and additionally they should have finite $(p, m)$-energy. The classes $\mathcal{E}_{p, m}(\Omega)$ are sometime known as the Cegrell's generalized energy classes, after Cegrell's influential work [15] on $\mathcal{E}_{p, n}(\Omega)$. For the early work on the theory of variation for the complex $n$-Hessian operator, see, e.g., $[9,10,18,23,24,28]$. On the other hand, if $m=1$, and $p=1$, then 
$e_{1,1}$ is the Dirichlet energy integral from potential theory connected to a long and fruitful history.

Set $X=\mathcal{E}_{p, m}(\Omega)$, and let $\mathrm{J}_{p}: X \times X \rightarrow[0, \infty)$ be defined by

$$
\mathrm{J}_{p}(u, v)=\left(\int|u-v|^{p}\left(\mathrm{H}_{m}(u)+\mathrm{H}_{m}(v)\right)\right)^{\frac{1}{p+m}} .
$$

In Theorem 3.6, we prove that $\left(X, \mathrm{~J}_{p}\right)$ is a quasimetric space in the above sense, and in Theorem 3.9, we prove that it is complete. Later in Sect. 7, we shall consider the compact Kähler manifold case, and in Theorems 3.6, and 7.5, we shall prove that the corresponding construction is a complete quasimetric space. Guedj et al. [25, Theorem 1.6] proved the quasi-triangle inequality in the case $m=n$, in the compact Kähler manifold setting (see also [11, Theorem 1.8], and [22]).

In Sect. 3, we will use the complete quasimetric space $\left(X, \mathrm{~J}_{p}\right)$ in $\mathbb{C}^{n}$ to prove the following stability results for the complex Hessian operators. First, let us define

$$
\begin{gathered}
\mathcal{M}_{p, m}=\{\mu: \mu \text { is a non-negative Radon measure on } \Omega \text { such that } \\
\left.\mathrm{H}_{m}(u)=\mu \text { for some } u \in \mathcal{E}_{p, m}(\Omega)\right\} .
\end{gathered}
$$

Let $\mu \in \mathcal{M}_{p, m}$, then in Theorem 6.3, we prove that if $0 \leq f, f_{j} \leq 1$ are measurable functions such that $f_{j} \rightarrow f$ in $L_{l o c}^{1}(\mu)$, as $j \rightarrow \infty$, then $\mathrm{J}_{p}\left(U\left(f_{j} \mu\right), U(f \mu)\right) \rightarrow 0$, $j \rightarrow \infty$. By Proposition 4.2 we know that convergence in $\left(X, \mathrm{~J}_{p}\right)$ implies convergence in capacity, but by Example 4.3, we have that the converse statement is false. Hence, Theorem 6.3 is a generalization of [34, Theorem 7.2]. Note that this also implies improved results in the pluricomplex case, $m=n$, and therefore, Theorem 6.3 also generalizes the stability result by Cegrell and Kołodziej [16]. For further information about these types of stability results in the case $m=n$, we refer to [19, Section 7.2].

We would like to thank the referees for their helpful comments and suggestions.

\section{Preliminaries}

Here, we shall present some crucial and necessary facts about $m$-subharmonic functions that shall be used in this paper. For further information, see, e.g., [1,2,29]. First, let $n \geq 2,1 \leq m \leq n$, and let $\Omega$ be a bounded domain in $\mathbb{C}^{n}$. Then define $\mathbb{C}_{(1,1)}$ to be the set of $(1,1)$-forms with constant coefficients, and set

$$
\Gamma_{m}=\left\{\alpha \in \mathbb{C}_{(1,1)}: \alpha \wedge \beta^{n-1} \geq 0, \ldots, \alpha^{m} \wedge \beta^{n-m} \geq 0\right\}
$$

where $\beta=d d^{c}|z|^{2}$ is the canonical Kähler form in $\mathbb{C}^{n}$. We then say that a subharmonic function $u$ defined on $\Omega$ is $m$-subharmonic, if the following inequality holds

$$
d d^{c} u \wedge \alpha_{1} \wedge \cdots \wedge \alpha_{m-1} \wedge \beta^{n-m} \geq 0
$$


in the sense of currents for all $\alpha_{1}, \ldots, \alpha_{m-1} \in \Gamma_{m}$. Furthermore, we call $\Omega$ for $m$ hyperconvex if it admits an exhaustion function $\varphi$ that is negative and $m$-subharmonic, i.e., the closure of the set $\{z \in \Omega: \varphi(z)<c\}$ is compact in $\Omega$, for every $c \in(-\infty, 0)$. For further information about $m$-hyperconvex domains, we refer to [5].

Let $p>0$. We say that an $m$-subharmonic function $\varphi$ defined on an $m$-hyperconvex domains $\Omega$ belongs to:

(i) $\mathcal{E}_{0, m}(\Omega)$ if, $\varphi$ is bounded,

$$
\lim _{z \rightarrow \xi} \varphi(z)=0 \quad \text { for every } \xi \in \partial \Omega
$$

and

$$
\int_{\Omega} \mathrm{H}_{m}(\varphi)<\infty
$$

where $\mathrm{H}_{m}(u)=\left(d d^{c} u\right)^{m} \wedge \beta^{n-m}$ is the complex Hessian operator.

(ii) $\mathcal{E}_{p, m}(\Omega)$ if, there exists a decreasing sequence, $\left\{u_{j}\right\}, u_{j} \in \mathcal{E}_{0, m}(\Omega)$, that converges pointwise to $u$ on $\Omega$, as $j$ tends to $\infty$, and

$$
\sup _{j} e_{p, m}\left(u_{j}\right)=\sup _{j} \int_{\Omega}\left(-u_{j}\right)^{p} \mathrm{H}_{m}\left(u_{j}\right)<\infty \text {. }
$$

Theorem 2.1 Let $n \geq 2,1 \leq m \leq n$, and assume that $\Omega \subset \mathbb{C}^{n}$ is an m-hyperconvex domain. There exists a constant $D(m, p)$ (depending only on $p$ and $m$ ) such that for any $u_{0}, u_{1}, \ldots, u_{m} \in \mathcal{E}_{p, m}(\Omega)$ it holds

$$
\begin{aligned}
& \int_{\Omega}\left(-u_{0}\right)^{p} d d^{c} u_{1} \wedge \cdots \wedge d d^{c} u_{m} \wedge \beta^{n-m} \\
& \quad \leq D(m, p) e_{p, m}\left(u_{0}\right)^{\frac{p}{m+p}} e_{p, m}\left(u_{1}\right)^{\frac{1}{m+p}} \cdots e_{p, m}\left(u_{m}\right)^{\frac{1}{m+p}} .
\end{aligned}
$$

Proof See, e.g., Lu [29,30], and Nguyễn [33]. For the case when $m=n$, see [3,15,17, 35].

Theorem 2.2 Let $n \geq 2,1 \leq m \leq n$, and assume that $\Omega \subset \mathbb{C}^{n}$ is an m-hyperconvex domain. Furthermore, assume that $u, v \in \mathcal{E}_{p, m}(\Omega)$, and $T$ be a positive closed current. Then it holds:

$$
\int_{\{u<v\}} \mathrm{H}_{m}(v) \leq \int_{\{u<v\}} \mathrm{H}_{m}(u) .
$$

(2) If $\mathrm{H}_{m}(v) \leq \mathrm{H}_{m}(u)$, then $u \leq v$.

(3) If $\mathrm{H}_{m}(u)(u<v)=0$, then $u \geq v$.

(4) $\chi_{\{u<v\}}\left(d d^{c} \max (u, v)\right) \wedge T=\chi_{\{u<v\}}\left(d d^{c} v\right) \wedge T$.

(5) $\mathrm{H}_{m}(\max (u, v)) \geq \chi\{u \geq v\} \mathrm{H}_{m}(u)+\chi_{\{u<v\}} \mathrm{H}_{m}(v)$. 
Proof For (1), (2), (4), and (5), see, e.g., [30,34]. Point (2) was proved in [8] for $p=1$. The proof for $p \neq 1$ is the same.

We shall need a comparison principle with weights. Proposition 2.3 will be used in the proof of Proposition 3.4.

Proposition 2.3 Let $n \geq 2,1 \leq m \leq n$, and assume that $\Omega \subset \mathbb{C}^{n}$ is an $m$-hyperconvex domain. Assume that $u, v, w \in \mathcal{E}_{p, m}(\Omega)$ are such that $w \geq u \geq v$, then

$$
\int_{\Omega}(w-u)^{p} \mathrm{H}_{m}(u) \leq(\max (p, 1)+1)^{m} \int_{\Omega}(w-v)^{p} \mathrm{H}_{m}(v) .
$$

Proof Let $u_{1}=u-w, v_{1}=v-w$ and $T=\left(d d^{c} w+d d^{c} u_{1}\right)^{m-1} \wedge \beta^{n-m}$. Then we have

$$
\begin{aligned}
& \int_{\Omega}\left(-v_{1}\right)^{p}\left(d d^{c} w+d d^{c} u_{1}\right) \wedge T \\
& \quad=\int_{\Omega}\left(-v_{1}\right)^{p} d d^{c} w \wedge T+\int_{\Omega}\left(-v_{1}\right)^{p} d d^{c} u_{1} \wedge T=I_{1}+I_{2} .
\end{aligned}
$$

Note that for $p \geq 1$

$$
\begin{aligned}
d d^{c}\left(-\left(-v_{1}\right)^{p}\right) & =p(1-p)\left(-v_{1}\right)^{p-2} d v_{1} \wedge d^{c} v_{1}+p\left(-v_{1}\right)^{p-1} d d^{c} v_{1} \\
& \leq p\left(-v_{1}\right)^{p-1}\left(d d^{c} v_{1}+d d^{c} w\right)
\end{aligned}
$$

and for $p<1$

$$
\begin{aligned}
d d^{c}\left(-\left(-v_{1}\right)^{p}\right) & =p(1-p)\left(-v_{1}\right)^{p-2} d v_{1} \wedge d^{c} v_{1}+p\left(-v_{1}\right)^{p-1} d d^{c} v_{1} \\
& \leq p(1-p)\left(-v_{1}\right)^{p-2} d v_{1} \wedge d^{c} v_{1}+p\left(-v_{1}\right)^{p-1}\left(d d^{c} v_{1}+d d^{c} w\right)
\end{aligned}
$$

Then we get

$$
\begin{aligned}
I_{1}= & \int_{\Omega}\left(-v_{1}\right)^{p} d d^{c} w \wedge T \leq \int_{\Omega}\left(-v_{1}\right)^{p} d d^{c} w \wedge T \\
& +p \int_{\Omega}\left(-v_{1}\right)^{p-1} d v_{1} \wedge d^{c} v_{1} \wedge T=\int_{\Omega}\left(-v_{1}\right)^{p}\left(d d^{c} w+d d^{c} v_{1}\right) \wedge T .
\end{aligned}
$$

For $p \geq 1$

$$
\begin{aligned}
I_{2} & =\int_{\Omega}\left(-v_{1}\right)^{p} d d^{c} u_{1} \wedge T=\int_{\Omega}\left(-u_{1}\right) d d^{c}\left(-\left(-v_{1}\right)^{p}\right) \wedge T \\
& \leq p \int_{\Omega}\left(-u_{1}\right)\left(-v_{1}\right)^{p-1}\left(d d^{c} v_{1}+d d^{c} w\right) \wedge T \leq p \int_{\Omega}\left(-v_{1}\right)^{p}\left(d d^{c} v_{1}+d d^{c} w\right) \wedge T,
\end{aligned}
$$


and for $p<1$

$$
\begin{aligned}
I_{2} & =\int_{\Omega}\left(-v_{1}\right)^{p} d d^{c} u_{1} \wedge T=\int_{\Omega}\left(-u_{1}\right) d d^{c}\left(-\left(-v_{1}\right)^{p}\right) \wedge T \\
& \leq \int_{\Omega}\left(-u_{1}\right)\left(p(1-p)\left(-v_{1}\right)^{p-2} d v_{1} \wedge d^{c} v_{1}+p\left(-v_{1}\right)^{p-1}\left(d d^{c} v_{1}+d d^{c} w\right)\right) \wedge T \\
& \leq \int_{\Omega} p(1-p)\left(-v_{1}\right)^{p-1} d v_{1} \wedge d^{c} v_{1} \wedge T+p\left(-v_{1}\right)^{p}\left(d d^{c} v_{1}+d d^{c} w\right) \wedge T \\
& \leq(1-p) \int_{\Omega}\left(-v_{1}\right)^{p} d d^{c} v_{1} \wedge T+p \int_{\Omega}\left(-v_{1}\right)^{p}\left(d d^{c} v_{1}+d d^{c} w\right) \wedge T \\
& \leq \int_{\Omega}\left(-v_{1}\right)^{p}\left(d d^{c} v_{1}+d d^{c} w\right) \wedge T .
\end{aligned}
$$

Finally, for any $p>0$

$$
\begin{aligned}
& \int_{\Omega}\left(-u_{1}\right)^{p}\left(d d^{c} w+d d^{c} u_{1}\right) \wedge T \leq \int_{\Omega}\left(-v_{1}\right)^{p}\left(d d^{c} w+d d^{c} u_{1}\right) \wedge T \\
& \quad \leq(\max (p, 1)+1) \int_{\Omega}\left(-v_{1}\right)^{p}\left(d d^{c} w+d d^{c} v_{1}\right) \wedge T \leq \ldots \\
& \quad \leq(\max (p, 1)+1)^{m} \int_{\Omega}(w-v)^{p}\left(d d^{c} v\right)^{m} \wedge \beta^{n-m} .
\end{aligned}
$$

We end this section with a Xing tyle inequality, see [37], that shall be used in Proposition 3.4. In Proposition 7.3, we shall as well prove the correspondent result in the case of compact Kähler manifolds.

Proposition 2.4 Let $n \geq 2,1 \leq m \leq n$, and assume that $\Omega \subset \mathbb{C}^{n}$ is an $m$-hyperconvex domain, and $u, v \in \mathcal{E}_{p, m}(\Omega)$.

(1) If $u \leq v$, then

$$
\int_{\Omega}(v-u)^{p} \mathrm{H}_{m}(v) \leq \int_{\Omega}(v-u)^{p} \mathrm{H}_{m}(u) .
$$

(2) Without any additional assumption on $u$, and $v$, it holds

$$
\int_{\{u<v\}}(v-u)^{p} \mathrm{H}_{m}(v) \leq \int_{\{u<v\}}(v-u)^{p} \mathrm{H}_{m}(u) .
$$

Proof (1) Let $\epsilon>1$, then $\epsilon u<u \leq v$. We obtain

$$
\begin{aligned}
& \int_{\Omega}(v-\epsilon u)^{p}\left(\mathrm{H}_{m}(\epsilon u)-\mathrm{H}_{m}(v)\right) \\
& =\sum_{k+l=m-1} \int_{\Omega}(v-\epsilon u)^{p} d d^{c}(\epsilon u-v) \wedge\left(d d^{c} \epsilon u\right)^{k} \wedge\left(d d^{c} v\right)^{l} \wedge \beta^{n-m}
\end{aligned}
$$




$$
\begin{aligned}
& =p \sum_{k+l=m-1} \int_{\Omega}(v-\epsilon u)^{p-1} d(v-\epsilon u) \wedge d^{c}(v-\epsilon u) \wedge\left(d d^{c} \epsilon u\right)^{k} \wedge\left(d d^{c} v\right)^{l} \wedge \beta^{n-m} \\
& \geq 0 .
\end{aligned}
$$

From this, it follows

$$
\int_{\Omega}(v-\epsilon u)^{p} \mathrm{H}_{m}(v) \leq \epsilon^{m} \int_{\Omega}(v-\epsilon u)^{p} \mathrm{H}_{m}(u),
$$

and then by using the monotone convergence theorem, and finally passing to the limit, $\epsilon \rightarrow 1^{+}$, we arrive at the desired conclusion.

(2) From (1), and Theorem 2.2, we obtain

$$
\begin{aligned}
& \int_{\{u<v\}}(v-u)^{p} \mathrm{H}_{m}(v)=\int_{\{u<v\}}(\max (u, v)-u)^{p} \mathrm{H}_{m}(\max (u, v)) \\
& =\int_{\Omega}(\max (u, v)-u)^{p} \mathrm{H}_{m}(\max (u, v)) \leq \int_{\Omega}(\max (u, v)-u)^{p} \mathrm{H}_{m}(u) \\
& =\int_{\{u<v\}}(v-u)^{p} \mathrm{H}_{m}(u)
\end{aligned}
$$

\section{Quasimetric Spaces}

Let $(X, d)$ be a quasimetric space. Recall that every metric is a quasimetric. Furthermore, in every quasimetric space $(X, d)$ there exists a metric $\rho$ with the property that there is an $\epsilon>0$, and a constant $A>0$ such that

$$
A^{-1} d^{\epsilon} \leq \rho \leq A d^{\epsilon}
$$

see, e.g., [26].

In the next definition, we shall define a functional, $\mathrm{J}_{p}$, in $\mathcal{E}_{p, m}(\Omega) \times \mathcal{E}_{p, m}(\Omega)$. After proving some elementary properties of $\mathrm{J}_{p}$ in Proposition 3.4 , and that $\mathrm{J}_{p}$ satisfies the quasi-triangle inequality (Lemma 3.5), we can, in Theorem 3.6, conclude that we have a family of quasimetric spaces. These spaces are complete as shall be shown in Theorem 3.9.

Definition 3.1 Let $n \geq 2,1 \leq m \leq n$, and assume that $\Omega \subset \mathbb{C}^{n}$ is an $m$-hyperconvex domain. For $u, v \in \mathcal{E}_{p, m}(\Omega)$ and $p>0$ let us define

$$
\mathrm{J}_{p}(u, v)=\left(\int_{\Omega}|u-v|^{p}\left(\mathrm{H}_{m}(u)+\mathrm{H}_{m}(v)\right)\right)^{\frac{1}{p+m}} .
$$

In the next definition, let us recall the notion of rooftop envelope. 
Definition 3.2 Let $n \geq 2,1 \leq m \leq n$, and assume that $\Omega \subset \mathbb{C}^{n}$ is an $m$-hyperconvex domain. For $u_{1}, \ldots, u_{k} \in \mathcal{E}_{p, m}(\Omega)$ define

$$
\mathrm{P}\left(u_{1}, \ldots, u_{k}\right)=\left(\sup \left\{\varphi \in \mathcal{E}_{p, m}(\Omega): \varphi \leq \min \left(u_{1}, \ldots, u_{k}\right)\right\}\right)^{*},
$$

where ()$^{*}$ is the upper semicontinuous regularization.

Remark If $u, v \in \mathcal{E}_{p, m}(\Omega)$, then $u+v \leq \mathrm{P}(u, v)$, and therefore we have $P(u, v) \in$ $\mathcal{E}_{p, m}(\Omega)$.

We shall need the following minimum principle. In [8, Theorem 4.3], Theorem 3.3 was proved for the class $\mathcal{E}_{1, m}(\Omega)$, but the proof without any change goes over to $\mathcal{E}_{p, m}(\Omega)$. Therefore, we omit the proof here.

Theorem 3.3 Let $n \geq 2,1 \leq m \leq n$, and assume that $\Omega \subset \mathbb{C}^{n}$ is an m-hyperconvex domain. Let $u, v \in \mathcal{E}_{p, m}(\Omega)$. Then the following holds

$$
\mathrm{H}_{m}(\mathrm{P}(u, v)) \leq \chi_{\{\mathrm{P}(u, v)=u\}} \mathrm{H}_{m}(u)+\chi_{\{\mathrm{P}(u, v)=v\}} \mathrm{H}_{m}(v) .
$$

Proposition 3.4 Let $n \geq 2,1 \leq m \leq n$, and assume that $\Omega \subset \mathbb{C}^{n}$ is an $m$-hyperconvex domain. Furthermore assume that $u, v, w \in \mathcal{E}_{p, m}(\Omega)$. Then

(1) $\mathrm{J}_{p}(u, v)<\infty$;

(2) $\mathrm{J}_{p}(u, v)=0$ if, and only if, $u=v$;

(3) $\mathrm{J}_{p}(u, v)=\mathrm{J}_{p}(v, u)$;

(4) $\mathrm{J}_{p}(u, v)^{p+m}=\mathrm{J}_{p}(u, \max (u, v))^{p+m}+\mathrm{J}_{p}(v, \max (u, v))^{p+m}$;

$$
\begin{aligned}
& \max \left(\mathrm{J}_{p}(u, \max (u, v)), \mathrm{J}_{p}(v, \max (u, v))\right) \leq \mathrm{J}_{p}(u, v) \\
& \quad \leq \mathrm{J}_{p}(u, \max (u, v))+\mathrm{J}_{p}(v, \max (u, v)) ;
\end{aligned}
$$

(6) If $u \leq v$, then

$$
2 \int_{\Omega}(v-u)^{p} \mathrm{H}_{m}(v) \leq \mathrm{J}_{p}(u, v)^{p+m} \leq 2 \int_{\Omega}(v-u)^{p} \mathrm{H}_{m}(u) ;
$$

(7) If $u \leq v \leq w$, then $\mathrm{J}_{p}(u, v) \leq 2^{\frac{p+2}{p+m}} \mathrm{~J}_{p}(u, w)$;

(8) If $u \leq v \leq w$, then $\mathrm{J}_{p}(v, w)^{p+m} \leq(\max (p, 1)+1)^{m} \mathrm{~J}_{p}(u, w)^{p+m}$;

(9) $\mathrm{J}_{p}(v, \mathrm{P}(u, v)) \leq \mathrm{J}_{p}(u, \max (u, v)) \leq \mathrm{J}_{p}(u, v)$;

(10) $\mathrm{J}_{p}(v, \mathrm{P}(u, v))^{p+m}+\mathrm{J}_{p}(u, \mathrm{P}(u, v))^{p+m} \leq \mathrm{J}_{p}(v, u)^{p+m}$;

(11) If $u \leq v$, then $\mathrm{J}_{p}(\mathrm{P}(u, w), \mathrm{P}(v, w)) \leq 2^{\frac{p+2}{p+m}} \mathrm{~J}_{p}(u, v)$.

Proof (1). By Theorem 2.1 we have

$$
\begin{aligned}
\mathrm{J}_{p}(u, v)^{p+m} & \leq \int_{\Omega}(-u-v)^{p}\left(\mathrm{H}_{m}(u)+\mathrm{H}_{m}(v)\right) \\
& \leq D(m, p) e_{p, m}(u+v)^{\frac{p}{p+m}}\left(e_{p}(u)^{\frac{m}{p+m}}+e_{p}(v)^{\frac{m}{p+m}}\right)<\infty .
\end{aligned}
$$


(2). It is obvious that $\mathrm{J}_{p}(u, u)=0$. Next, assume that $\mathrm{J}_{p}(u, v)=0$. Then $\mathrm{H}_{m}(u)(\{u<$ $v\})=0$, so by Theorem 2.2 we obtain $u \geq v$. In a similar manner, we have $\mathrm{H}_{m}(v)(\{v<$ $u\})=0$. Hence, $v \leq u$, and therefore it follows $u=v$.

(3). This property is an immediate consequence of the definition of $\mathbf{J}_{p}$.

(4). Thanks to Theorem 2.2, it follows that $\mathrm{H}_{m}(\max (u, v))=\mathrm{H}_{m}(u)$ on the set $\{u>v\}$, and similarly $\left.\mathrm{H}_{m}(\max (u, v))\right)=\mathrm{H}_{m}(v)$ on the set $\{v>u\}$. Thus,

$$
\begin{aligned}
\mathrm{J}_{p}(u, v)^{p+m}= & \int_{\Omega}|u-v|^{p}\left(\mathrm{H}_{m}(u)+\mathrm{H}_{m}(v)\right) \\
= & \int_{\{u<v\}}(\max (u, v)-u)^{p}\left(\mathrm{H}_{m}(u)+\mathrm{H}_{m}(\max (u, v))\right) \\
& +\int_{\{v<u\}}(\max (u, v)-v)^{p}\left(\mathrm{H}_{m}(v)+\mathrm{H}_{m}(\max (u, v))\right) \\
= & \mathbf{J}_{p}(u, \max (u, v))^{p+m}+\mathrm{J}_{p}(v, \max (u, v))^{p+m} .
\end{aligned}
$$

(5). This is an immediate consequence of (4).

(6). Proposition 2.4 yields this result.

(7). Note that $0 \leq w-v \leq w-u$. Then by using (6) we get

$$
\begin{aligned}
\mathrm{J}_{p}(u, v)^{p+m} & \leq 2\left(\int_{\Omega}(v-u)^{p} \mathrm{H}_{m}(u)\right) \\
& \leq 2^{1+p}\left(\int_{\Omega}(w-v)^{p} \mathrm{H}_{m}(u)+\int_{\Omega}(w-u)^{p} \mathrm{H}_{m}(u)\right) \\
& \leq 2^{2+p} \mathbf{J}_{p}(u, w)^{p+m} .
\end{aligned}
$$

(8). By Proposition 2.3 we get

$$
\begin{aligned}
\mathrm{J}_{p}(v, w)^{p+m} & =\int_{\Omega}(w-v)^{p}\left(\mathrm{H}_{m}(v)+\mathrm{H}_{m}(w)\right) \\
& =\int_{\Omega}(w-v)^{p} \mathrm{H}_{m}(v)+\int_{\Omega}(w-v)^{p} \mathrm{H}_{m}(w) \\
& \leq(\max (p, 1)+1)^{m} \int_{\Omega}(w-u)^{p} \mathrm{H}_{m}(u)+\int_{\Omega}(w-u)^{p} \mathrm{H}_{m}(w) \\
& =(\max (p, 1)+1)^{m} \mathrm{~J}_{p}(u, w)^{p+m} .
\end{aligned}
$$

(9). By Theorem 2.2 we get

$$
\begin{aligned}
\mathrm{J}_{p}(u, \max (u, v))^{p+m} & =\int_{\Omega}(\max (u, v)-u)^{p}\left(\mathrm{H}_{m}(u)+\mathrm{H}_{m}(\max (u, v))\right) \\
& \left.=\int_{\{u<v\}}(v-u)^{p}\left(\mathrm{H}_{m}(u)+\mathrm{H}_{m}(\max (u, v))\right)\right) \\
& =\int_{\{u<v\}}(v-u)^{p}\left(\mathrm{H}_{m}(u)+\mathrm{H}_{m}(v)\right) .
\end{aligned}
$$


On the other hand, using Theorem 3.3 we arrive at

$$
\begin{aligned}
\mathrm{J}_{p}(v, \mathrm{P}(u, v))^{p+m}= & \int_{\Omega}(v-\mathrm{P}(u, v))^{p}\left(\mathrm{H}_{m}(v)+\mathrm{H}_{m}(\mathrm{P}(u, v))\right) \\
\leq & \int_{\{\mathrm{P}(u, v)<v\}}(v-\mathrm{P}(u, v))^{p}\left(\mathrm{H}_{m}(v)+\chi\{\mathrm{P}(u, v)=v\}\right. \\
& \quad+\chi\{\mathrm{P}(u, v)=u\} \\
& \left.\left.\mathrm{H}_{m}(v)\right)\right) \\
= & \int_{\{\mathrm{P}(u, v)<v\} \cap\{\mathrm{P}(u, v)=u\}}(v-\mathrm{P}(u, v))^{p}\left(\mathrm{H}_{m}(u)+\mathrm{H}_{m}(v)\right) \\
\leq & \int_{\{u<v\}}(v-u)^{p}\left(\mathrm{H}_{m}(u)+\mathrm{H}_{m}(v)\right)=\mathrm{J}_{p}(u, \max (u, v))^{p+m} .
\end{aligned}
$$

The last inequality follows from (5).

(10). This follows from (4) together with (9).

(11). Note that $u \leq \max (u, \mathrm{P}(v, w)) \leq v$, and then by (7) and (9), we have

$$
\begin{aligned}
\mathrm{J}_{p}(\mathrm{P}(v, w), \mathrm{P}(u, w)) & =\mathrm{J}_{p}(\mathrm{P}(v, w), \mathrm{P}(u, \mathrm{P}(v, w))) \leq \mathrm{J}_{p}(u, \max (u, \mathrm{P}(v, w))) \\
& \leq 2^{\frac{p+2}{p+m}} \mathbf{J}_{p}(u, v) .
\end{aligned}
$$

By letting us be inspired by [25], we can in the next lemma prove that $\mathrm{J}_{p}$ enjoys the quasi-triangle inequality.

Lemma 3.5 Let $n \geq 2,1 \leq m \leq n$, and assume that $\Omega \subset \mathbb{C}^{n}$ is an $m$-hyperconvex domain. Then there exists $C>0$ such that for any $u, v, w \in \mathcal{E}_{p, m}(\Omega)$ it holds

$$
\mathrm{J}_{p}(u, v) \leq C\left(\mathrm{~J}_{p}(u, w)+\mathrm{J}_{p}(w, v)\right)
$$

Furthermore, the constant $C$ can be taken as $C=\left(2^{2 p+1}\left(2^{p}+1\right) 3^{m}\right)^{\frac{1}{p+m}}$.

Proof By using the comparison principle (see, e.g., Theorem 2.2), it follows

$$
\begin{aligned}
& \mathrm{H}_{m}(u)(\{v<u-2 s\}) \leq \mathrm{H}_{m}(v)(\{v<u-2 s\}) \\
& \mathrm{H}_{m}(v)(\{u<v-2 s\}) \leq \mathrm{H}_{m}(u)(\{u<v-2 s\}),
\end{aligned}
$$

and therefore it holds

$$
\begin{aligned}
\mathrm{J}_{p}(u, v)^{p+m} & =\int_{\Omega}|u-v|^{p}\left(\mathrm{H}_{m}(u)+\mathrm{H}_{m}(v)\right) \\
& =p \int_{0}^{\infty} s^{p-1}\left(\mathrm{H}_{m}(u)+\mathrm{H}_{m}(v)\right)(\{|u-v|>s\}) \mathrm{d} s
\end{aligned}
$$




$$
\begin{aligned}
& =p 2^{p} \int_{0}^{\infty} s^{p-1}\left(\mathrm{H}_{m}(u)+\mathrm{H}_{m}(v)\right)(\{|u-v|>2 s\}) \mathrm{d} s \\
& \leq p 2^{p+1} \int_{0}^{\infty} s^{p-1}\left(\mathrm{H}_{m}(u)(\{u<v-2 s\})+\mathrm{H}_{m}(v)(\{v<u-2 s\})\right) \mathrm{d} s .
\end{aligned}
$$

Next we shall estimate the measure $\mathrm{H}_{m}(u)(\{u<v-2 s\})$. Since

$$
\begin{aligned}
\{u<v-2 s\} & \subset\{u<w-s\} \cup\{w-s \leq u<v-2 s\} \subset\{u<w-s\} \\
\cup & \left\{w<\frac{u+2 v}{3}-\frac{s}{3}\right\}
\end{aligned}
$$

we can use again the comparison principle (see, e.g., Theorem 2.2) and arrive at

$$
\begin{aligned}
& \mathrm{H}_{m}(u)(\{w-s \leq u<v-2 s\}) \\
& \quad \leq \mathrm{H}_{m}(u)\left(\left\{w<\frac{u+2 v}{3}-\frac{s}{3}\right\}\right) \\
& \quad \leq 3^{m} \mathrm{H}_{m}\left(\frac{u+2 v}{3}\right)\left(\left\{w<\frac{u+2 v}{3}-\frac{s}{3}\right\}\right) \\
& \quad \leq 3^{m} \mathrm{H}_{m}(w)\left(\left\{w<\frac{u+2 v}{3}-\frac{s}{3}\right\}\right) .
\end{aligned}
$$

Note that also holds

$$
\begin{aligned}
\left|w-\frac{u+2 v}{3}\right|^{p} & =\frac{1}{3^{p}}|3 w-u-2 v|^{p} \leq\left(\frac{2}{3}\right)^{p}\left(|w-u|^{p}+|2 w-2 v|^{p}\right) \\
& \leq\left(\frac{2}{3}\right)^{p}|w-u|^{p}+\left(\frac{4}{3}\right)^{p}|w-v|^{p} .
\end{aligned}
$$

Finally, by using the above estimates

$$
\begin{aligned}
& p \int_{0}^{\infty} s^{p-1} \mathrm{H}_{m}(u)(\{u<v-2 s\}) \mathrm{d} s \\
& \leq p \int_{0}^{\infty} s^{p-1} \mathrm{H}_{m}(u)(\{u<w-s\}) \mathrm{d} s \\
& \quad+p 3^{m} \int_{0}^{\infty} s^{p-1} \mathrm{H}_{m}(w)\left(\left\{w<\frac{u+2 v}{3}-\frac{s}{3}\right\}\right) \mathrm{d} s \\
& \leq \int_{\Omega}|w-u|^{p} \mathrm{H}_{m}(u)+3^{m+p} \int_{\Omega}\left|w-\frac{u+2 v}{3}\right|^{p} \mathrm{H}_{m}(w) \\
& \leq \int_{\Omega}|w-u|^{p} \mathrm{H}_{m}(u)+3^{m} 2^{p} \int_{\Omega}|w-u|^{p} \mathrm{H}_{m}(w)+3^{m} 4^{p} \int_{\Omega}|w-v|^{p} \mathrm{H}_{m}(w) .
\end{aligned}
$$


A similar estimate can be obtained for $\mathrm{H}_{m}(v)(\{v<u-2 s\})$, and therefore by (3.2) we get

$$
\begin{aligned}
\mathrm{J}_{p}(u, v)^{p+m} \leq & p 2^{p+1} \int_{0}^{\infty} s^{p-1}\left(\mathrm{H}_{m}(u)(\{u<v-2 s\})+\mathrm{H}_{m}(v)(\{v<u-2 s\})\right) \mathrm{d} s \\
\leq & 2^{p+1}\left(\int_{\Omega}|w-u|^{p} \mathrm{H}_{m}(u)+3^{m} 2^{p} \int_{\Omega}|w-u|^{p} \mathrm{H}_{m}(w)\right. \\
& \left.+3^{m} 4^{p} \int_{\Omega}|w-v|^{p} \mathrm{H}_{m}(w)\right) \\
& +2^{p+1}\left(\int_{\Omega}|w-v|^{p} \mathrm{H}_{m}(v)+3^{m} 2^{p} \int_{\Omega}|w-v|^{p} \mathrm{H}_{m}(w)\right. \\
& \left.+3^{m} 4^{p} \int_{\Omega}|w-u|^{p} \mathrm{H}_{m}(w)\right) \\
\leq & 2^{2 p+1}\left(2^{p}+1\right) 3^{m}\left(\mathrm{~J}_{p}(u, w)^{p+m}+\mathrm{J}_{p}(v, w)^{p+m}\right) .
\end{aligned}
$$

To finish the proof, it is enough to observe that

$$
\left.\mathrm{J}_{p}(u, v) \leq\left(2^{2 p+1}\left(2^{p}+1\right) 3^{m}\right)\right)^{\frac{1}{p+m}}\left(\mathbf{J}_{p}(u, w)+\mathbf{J}_{p}(v, w)\right)
$$

Thanks to Proposition 3.4 (1)-(3) and Lemma 3.5 we can now conclude that we have a family of quasimetric spaces. The aim of the rest of this section is to prove that they are complete, which we will do in Theorem 3.9.

Theorem 3.6 Let $n \geq 2,1 \leq m \leq n$, and assume that $\Omega \subset \mathbb{C}^{n}$ is an $m$-hyperconvex domain. Then the pair $\left(\mathcal{E}_{p, m}(\Omega), \mathrm{J}_{p}\right)$ is a quasimetric space.

To be able to prove that the quasimetric spaces are complete, we need information on how $\mathbf{J}_{p}$ behaves under monotone sequences. In the case $p=1, \mathbf{J}_{1}$ is continuous both for increasing and decreasing sequences, but only for decreasing sequences when $p \neq 1$.

Proposition 3.7 Let $n \geq 2,1 \leq m \leq n$, and assume that $\Omega \subset \mathbb{C}^{n}$ is an m-hyperconvex domain. Let $u_{j} \in \mathcal{E}_{p, m}(\Omega)$ be a decreasing sequence that converging to $u \in \mathcal{E}_{p, m}(\Omega)$. Then $\mathrm{J}_{p}\left(u_{j}, u\right) \rightarrow 0$, as $j \rightarrow \infty$. If $p=1$, then the same statement is true for increasing sequences.

Proof First assume that the sequence $u_{j}$ is decreasing. Then by Proposition 3.4 (6), and the monotone convergence theorem, we get

$$
\mathrm{J}_{p}\left(u_{j}, u\right)^{p+m} \leq 2 \int_{\Omega}\left(u_{j}-u\right)^{p} \mathrm{H}_{m}(u) \rightarrow 0,
$$


as $j \rightarrow \infty$. Now assume that $p=1$, and the sequence $u_{j}$ is increasing. By Proposition

2.7 from [8] and Proposition 3.4 (6), we get

$$
\mathrm{J}_{1}\left(u_{j}, u\right)^{1+m} \leq 2 \int_{\Omega}\left(u-u_{j}\right) \mathrm{H}_{m}\left(u_{j}\right) \rightarrow 0,
$$

as $j \rightarrow \infty$.

To prove that the space $\left(\mathcal{E}_{p, m}(\Omega), \mathrm{J}_{p}\right)$ is complete we shall need the following elementary fact.

Proposition 3.8 If $f_{j}$ is an increasing sequence of continuous functions defined on $X$ such that $f_{j} \nearrow f$, and $\mu_{j} \rightarrow \mu$ weakly, as $j \rightarrow \infty$, then

$$
\liminf _{j \rightarrow \infty} \int_{X} f_{j} d \mu_{j} \geq \int_{X} f d \mu
$$

Proof First we shall prove that if $\alpha$ is a lower continuous function then

$$
\int_{X} \alpha d \mu \leq \liminf _{j \rightarrow \infty} \int_{X} \alpha d \mu_{j}
$$

Let $\mathcal{C}_{0}(X) \ni g_{k} \nearrow \alpha$, then $\int_{X} g_{k} d \mu_{j} \leq \int_{X} \alpha d \mu_{j}$. Now by the weak convergence we get

$$
\int_{X} g_{k} d \mu=\lim _{j \rightarrow \infty} \int_{X} g_{k} d \mu_{j} \leq \liminf _{j \rightarrow \infty} \int_{X} \alpha d \mu_{j}
$$

and by monotone convergence theorem we obtain (3.3).

Fix $k$, and let $j \geq k$, then $\int_{X} f_{j} d \mu_{j} \geq \int_{X} f_{k} d \mu_{j}$. Therefore by (3.3)

$$
\liminf _{j \rightarrow \infty} \int_{X} f_{j} d \mu_{j} \geq \liminf _{j \rightarrow \infty} \int_{X} f_{k} d \mu_{j} \geq \int_{X} f_{k} d \mu .
$$

From the monotone convergence theorem, it now follows

$$
\liminf _{j \rightarrow \infty} \int_{X} f_{j} d \mu_{j} \geq \lim _{k \rightarrow \infty} \int_{X} f_{k} d \mu=\int_{X} f d \mu .
$$

The completeness of $\left(\mathcal{E}_{p, m}(\Omega), \mathrm{J}_{p}\right)$ is next on our agenda.

Theorem 3.9 Let $n \geq 2,1 \leq m \leq n$, and assume that $\Omega \subset \mathbb{C}^{n}$ is an m-hyperconvex domain. The quasimetric space $\left(\mathcal{E}_{p, m}(\Omega), \mathrm{J}_{p}\right)$ is complete. 
Proof Let $\left\{\varphi_{j}\right\} \subset \mathcal{E}_{p, m}(\Omega)$ be a Cauchy sequence. After choosing a subsequence, we may assume that $\mathrm{J}_{p}\left(\varphi_{j}, \varphi_{j+1}\right) \leq \frac{1}{3(2 C)^{j+3}}$ for $j \in \mathbb{N}$, where $C$ is the constant from quasi-triangle inequality. From [5, Theorem 5.2], it follows that for each $\varphi_{j}$ there exists a decreasing sequence of continuous functions $\mathcal{E}_{0, m}(\Omega) \cap \mathcal{C}(\bar{\Omega}) \ni \psi_{j}^{k} \searrow \varphi_{j}$, $k \rightarrow \infty$. Then we can choose $u_{j}=\psi_{j}^{k(j)}$ such that

$$
\mathrm{J}_{p}\left(u_{j}, \varphi_{j}\right) \leq \frac{1}{3(2 C)^{j+3}},
$$

see Proposition 3.7. Observe that $\left\{u_{j}\right\} \subset \mathcal{E}_{p, m}(\Omega)$ is also a Cauchy sequence. For each $j \in \mathbb{N}$, we have

$$
\begin{aligned}
\mathrm{J}_{p}\left(u_{j}, u_{j+1}\right) & \leq C^{2}\left(\mathrm{~J}_{p}\left(u_{j}, \varphi_{j}\right)+\mathrm{J}_{p}\left(\varphi_{j}, \varphi_{j+1}\right)+\mathrm{J}_{p}\left(\varphi_{j+1}, u_{j+1}\right)\right) \\
& \leq \frac{3 C^{2}}{3(2 C)^{j+3}} \leq \frac{1}{(2 C)^{j+1}} .
\end{aligned}
$$

From the quasi-triangle inequality together with (3.5), we get

$$
\begin{aligned}
\mathrm{J}_{p}\left(0, u_{j}\right) & \leq C \mathrm{~J}_{p}\left(0, u_{1}\right)+C^{2} \mathrm{~J}_{p}\left(u_{1}, u_{2}\right)+\cdots+C^{j} \mathrm{~J}_{p}\left(u_{j-1}, u_{j}\right) \\
& \leq C \mathrm{~J}_{p}\left(0, u_{1}\right)+\frac{C^{2}}{(2 C)^{2}}+\cdots+\frac{C^{j}}{(2 C)^{j}} \leq C \mathrm{~J}_{p}\left(0, u_{1}\right)+1 .
\end{aligned}
$$

Set $v_{j, k}=\max \left(u_{j}, \ldots, u_{k}\right)$, for $k \geq j$. Since $v_{j, k} \in \mathcal{E}_{p, m}(\Omega)$, we can use Proposition 3.4 (9) and (3.5) to arrive at

$$
\begin{aligned}
\mathrm{J}_{p}\left(u_{j}, v_{j, k}\right) & =\mathrm{J}_{p}\left(u_{j}, \max \left(u_{j}, v_{j+1, k}\right)\right) \leq \mathrm{J}_{p}\left(u_{j}, v_{j+1, k}\right) \\
& \leq C\left(\mathrm{~J}_{p}\left(u_{j}, u_{j+1}\right)+\mathrm{J}_{p}\left(u_{j+1}, v_{j+1, k}\right)\right) \\
& =C\left(\mathrm{~J}_{p}\left(u_{j}, u_{j+1}\right)+\mathrm{J}_{p}\left(u_{j+1}, \max \left(u_{j+1}, v_{j+2, k}\right)\right)\right) \\
& \leq C \mathrm{~J}_{p}\left(u_{j}, u_{j+1}\right)+C \mathrm{~J}_{p}\left(u_{j+1}, v_{j+2, k}\right) \\
& \leq C \mathrm{~J}_{p}\left(u_{j}, u_{j+1}\right)+C^{2} \mathrm{~J}_{p}\left(u_{j+1}, u_{j+2}\right)+C^{2} \mathrm{~J}_{p}\left(u_{j+2}, v_{j+2, k}\right) \leq \ldots \\
& \leq \sum_{l=0}^{k-j-1} C^{l+1} \mathrm{~J}_{p}\left(u_{j+l}, u_{j+l+1}\right) \\
& \leq \sum_{l=0}^{k-j-1} C^{l+1} \frac{1}{(2 C)^{j+l+1}} \leq \frac{1}{2^{j}} .
\end{aligned}
$$

From (3.6), and (3.7), it now follows

$$
\begin{aligned}
\mathrm{J}_{p}\left(0, v_{j, k}\right) & \leq C\left(\mathrm{~J}_{p}\left(0, u_{j}\right)+\mathrm{J}_{p}\left(u_{j}, u_{v_{j, k}}\right)\right) \\
& \leq C\left(C \mathrm{~J}_{p}\left(0, u_{1}\right)+1+\frac{1}{2^{j}}\right) \leq C^{2}\left(\mathbf{J}_{p}\left(u_{1}, 0\right)+2\right) .
\end{aligned}
$$


The sequence $v_{j, k}$ is increasing in $k$, and therefore, it follows from (3.8) that $\sup _{k} e_{p, m}\left(v_{j, k}\right)<\infty$. Hence, $v_{j}=\left(\lim _{k \rightarrow \infty} v_{j, k}\right)^{*} \in \mathcal{E}_{p, m}(\Omega)$. Furthermore, $v_{j}$ is a decreasing sequence, $v_{j} \searrow u=\left(\lim \sup _{j \rightarrow \infty} u_{j}\right)^{*}$. Again using (3.8), we can conclude $\sup _{j} e_{p, m}\left(v_{j}\right)<\infty$. Thus, $u \in \mathcal{E}_{p, m}(\Omega)$.

We do not know if the quasimetric $\mathrm{J}_{p}$ is continuous under increasing sequences, but instead, we can obtain some estimates. The monotone convergence theorem and Proposition 3.8 imply

$$
\begin{aligned}
\liminf _{k \rightarrow \infty} \mathrm{J}_{p}\left(u_{j}, v_{j, k}\right)^{p+m}= & \liminf _{k \rightarrow \infty} \int_{\Omega}\left(v_{j, k}-u_{j}\right)^{p} \mathrm{H}_{m}\left(v_{j, k}\right) \\
& +\lim _{k \rightarrow \infty} \int_{\Omega}\left(v_{j, k}-u_{j}\right)^{p} \mathrm{H}_{m}\left(u_{j}\right) \geq \int_{\Omega}\left(v_{j}-u_{j}\right)^{p} \mathrm{H}_{m}\left(v_{j}\right) \\
& +\int_{\Omega}\left(v_{j}-u_{j}\right)^{p} \mathrm{H}_{m}\left(u_{j}\right) \\
= & \mathrm{J}_{p}\left(u_{j}, v_{j}\right)^{p+m}
\end{aligned}
$$

Hence, $\mathrm{J}_{p}\left(u_{j}, v_{j}\right) \rightarrow 0$, as $j \rightarrow \infty$, by using (3.7) and (3.9). Finally, $\varphi_{j}$ tends to $u$ in the quasimetric $\mathbf{J}_{p}$, since Proposition 3.7, and (3.4) yields

$$
\mathbf{J}_{p}\left(\varphi_{j}, u\right) \leq C^{2}\left(\mathbf{J}_{p}\left(u_{j}, v_{j}\right)+\mathbf{J}_{p}\left(v_{j}, u\right)+\mathbf{J}_{p}\left(u_{j}, \varphi_{j}\right)\right) \rightarrow 0
$$

as $j \rightarrow \infty$.

\section{Convergence in the Space $\left(\mathcal{E}_{p, m}(\Omega), J_{p}\right)$}

In this section, we shall continue to study the convergence in $\left(\mathcal{E}_{p, m}(\Omega), \mathrm{J}_{p}\right)$. From Proposition 3.7, we know that quasimetric $\mathrm{J}_{p}$ is continuous under decreasing sequences, and if $p=1$, then we also know continuity under increasing sequences. A summary of this section is as follows:

(1) If $\mathrm{J}_{p}\left(u_{j}, u\right) \rightarrow 0$, as $j \rightarrow \infty$, then $u_{j} \rightarrow u$ in $L^{p+m}(\Omega)$ (Proposition 4.1).

(2) If $\mathrm{J}_{p}\left(u_{j}, u\right) \rightarrow 0$, as $j \rightarrow \infty$, then $u_{j} \rightarrow u$ in capacity cap $m$ (Proposition 4.2).

(3) The inverse implications of the above results are not, in general valid (Example 4.3).

(4) If $\mathrm{J}_{p}\left(u_{j}, u\right) \rightarrow 0$, as $j \rightarrow \infty$, then $\mathrm{H}_{m}\left(u_{j}\right) \rightarrow \mathrm{H}_{m}(u)$ weakly (Proposition 4.5).

Proposition 4.1 Let $n \geq 2,1 \leq m \leq n$, and assume that $\Omega \subset \mathbb{C}^{n}$ is an $m$-hyperconvex domain and $u_{j}, u \in \mathcal{E}_{p, m}(\Omega)$. If $\mathrm{J}_{p}\left(u_{j}, u\right) \rightarrow 0, j \rightarrow \infty$, then $u_{j} \rightarrow u, j \rightarrow \infty$, in $L^{p+m}(\Omega)$.

Proof Recall that $\mathcal{E}_{p, m}(\Omega) \subset L^{p+m}(\Omega)$ (see, e.g., [6]). Let $\varphi \in \mathcal{E}_{0, m}(\Omega)$ be such that $\mathrm{H}_{m}(\varphi)=d V_{2 n}$. Assume that $\mathrm{J}_{p}\left(u_{j}, u\right) \rightarrow 0$, as $j \rightarrow \infty$. By Proposition 3.4 (5), we get

$$
\mathrm{J}_{p}\left(\max \left(u_{j}, u\right), u_{j}\right) \leq \mathrm{J}_{p}\left(u_{j}, u\right), \text { and } \mathrm{J}_{p}\left(\max \left(u_{j}, u\right), u\right) \leq \mathrm{J}_{p}\left(u_{j}, u\right),
$$


which implies that

$$
\mathrm{J}_{p}\left(\max \left(u_{j}, u\right), u_{j}\right) \rightarrow 0, \quad \mathrm{~J}_{p}\left(\max \left(u_{j}, u\right), u\right) \rightarrow 0, j \rightarrow \infty
$$

Thanks to Błocki’s inequality (see, e.g., Lemma 3.4 in [34]), together with (4.1), we obtain

$$
\begin{aligned}
\int_{\Omega}\left|u_{j}-u\right|^{p+m} d V_{2 n}= & \int_{\Omega}\left|u_{j}-u\right|^{p+m} \mathrm{H}_{m}(\varphi) \\
= & \int_{\left\{u_{j}<u\right\}}\left(u-u_{j}\right)^{p+m} \mathrm{H}_{m}(\varphi)+\int_{\left\{u<u_{j}\right\}}\left(u_{j}-u\right)^{p+m} \mathrm{H}_{m}(\varphi) \\
= & \int_{\left\{u_{j}<u\right\}}\left(\max \left(u, u_{j}\right)-u_{j}\right)^{p+m} \mathrm{H}_{m}(\varphi) \\
& +\int_{\left\{u<u_{j}\right\}}\left(\max \left(u_{j}, u\right)-u\right)^{p+m} \mathrm{H}_{m}(\varphi) \\
\leq & \int_{\Omega}\left(\max \left(u, u_{j}\right)-u_{j}\right)^{p+m} \mathrm{H}_{m}(\varphi) \\
& +\int_{\Omega}\left(\max \left(u_{j}, u\right)-u\right)^{p+m} \mathrm{H}_{m}(\varphi) \\
\leq & (p+m) \ldots(p+1)\|\varphi\|_{\infty}^{m}\left(\int_{\Omega}\left(\max \left(u, u_{j}\right)-u_{j}\right)^{p} \mathrm{H}_{m}\left(u_{j}\right)\right. \\
& \left.+\int_{\Omega}\left(\max \left(u_{j}, u\right)-u\right)^{p} \mathrm{H}_{m}(u)\right) \\
\leq & (p+m) \ldots(p+1)\|\varphi\|_{\infty}^{m}\left(\mathrm{~J}_{p}\left(\max \left(u_{j}, u\right), u_{j}\right)^{p+m}\right. \\
& \left.+\mathrm{J}_{p}\left(\max \left(u_{j}, u\right), u\right)^{p+m}\right) \rightarrow 0,
\end{aligned}
$$

as $j \rightarrow \infty$.

With $u_{j} \rightarrow u$ in capacity cap $_{m}$, we mean that for any $K \Subset \Omega$, and any $\epsilon>0$, it holds

$$
\lim _{j \rightarrow \infty} \operatorname{cap}_{m}\left(K \cap\left\{z \in \Omega:\left|u_{j}(z)-u(z)\right|>\epsilon\right\}\right)=0
$$

where the capacity of a Borel set $B \Subset \Omega$ is defined by

$$
\operatorname{cap}_{m}(B)=\sup \left\{\int_{B} \mathrm{H}_{m}(\varphi): \varphi \in \mathcal{S H}_{m}(\Omega) ;-1 \leq \varphi \leq 0\right\}
$$

Proposition 4.2 Let $n \geq 2,1 \leq m \leq n$, and assume that $\Omega \subset \mathbb{C}^{n}$ is an $m$-hyperconvex domain, and $u_{j}, u \in \mathcal{E}_{p, m}(\Omega)$. If $\mathrm{J}_{p}\left(u_{j}, u\right) \rightarrow 0$, as $j \rightarrow \infty$, then $u_{j} \rightarrow$ u in capacity cap $_{m}$. 
Proof Set $\varphi_{j}=\left(\sup _{k \geq j} u_{j}\right)^{*}$. Then it follows $\varphi_{j} \searrow v$, and $\varphi_{j} \geq u_{j}$. Since $\varphi_{j}$ is decreasing sequence, we get from [30] that $\varphi_{j} \rightarrow v$ in capacity cap ${ }_{m}$. Theorem 2.1 yields

$$
\begin{aligned}
e_{p, m}\left(\varphi_{j}\right)^{\frac{1}{p+m}} & \leq D(p, m)^{\frac{1}{p}} e_{p, m}\left(u_{j}\right)^{\frac{1}{p+m}}=D(p, m)^{\frac{1}{p}} \mathbf{J}_{p}\left(u_{j}, 0\right) \\
& \leq D(p, m)^{\frac{1}{p}} C\left(\mathbf{J}_{p}\left(u_{j}, u\right)+\mathbf{J}_{p}(u, 0)\right)
\end{aligned}
$$

which means that

$$
\sup _{j} e_{p, m}\left(\varphi_{j}\right)^{\frac{1}{p+m}} \leq D(p, m)^{\frac{1}{p}} \sup _{j} e_{p, m}\left(u_{j}\right)^{\frac{1}{p+m}}<\infty .
$$

Hence, $v \in \mathcal{E}_{p, m}(\Omega)$. Moreover, by Proposition 4.1, we know that $u_{j} \rightarrow u$ in $L^{p+m}(\Omega), j \rightarrow \infty$, and therefore also $\varphi_{j} \rightarrow u$ in $L^{p+m}(\Omega)$. This implies that $u=v$. We have by Proposition 3.7

$$
\mathrm{J}_{p}\left(u_{j}, \varphi_{j}\right) \leq C\left(\mathrm{~J}_{p}\left(u_{j}, u\right)+\mathrm{J}_{p}\left(\varphi_{j}, u\right)\right) \rightarrow 0,
$$

Now observe that

$$
\begin{aligned}
& \left\{z \in \Omega:\left|u_{j}(z)-u(z)\right|>\epsilon\right\} \subset \\
& \quad\left\{z \in \Omega:\left|\varphi_{j}(z)-u_{j}(z)\right|>\frac{\epsilon}{2}\right\} \cup\left\{z \in \Omega:\left|\varphi_{j}(z)-u(z)\right|>\frac{\epsilon}{2}\right\} .
\end{aligned}
$$

Therefore, it is sufficient to prove

$$
\lim _{j \rightarrow \infty} \operatorname{cap}_{m}\left(K \cap\left\{z \in \Omega:\left|\varphi_{j}(z)-u_{j}(z)\right|>\frac{\epsilon}{2}\right\}\right)=0 .
$$

Let $\psi \in \mathcal{E}_{0, m}(\Omega)$ be such that $-1 \leq \psi \leq 0$, and $K \Subset \Omega$. Then by Błocki's inequality (see, e.g., Lemma 3.4 in [34]) and by (4.2) we get

$$
\begin{aligned}
& \int_{K \cap\left\{z \in \Omega:\left|\varphi_{j}(z)-u_{j}(z)\right|>\frac{\epsilon}{2}\right\}} \mathrm{H}_{m}(\psi) \\
& \leq \frac{2^{m+p}}{\epsilon^{m+p}} \int_{K \cap\left\{z \in \Omega:\left|\varphi_{j}(z)-u_{j}(z)\right|>\frac{\epsilon}{2}\right\}}\left(\varphi_{j}-u_{j}\right)^{m+p} \mathrm{H}_{m}(\psi) \\
& \leq \frac{2^{m+p}}{\epsilon^{m+p}} \int_{\Omega}\left(\varphi_{j}-u_{j}\right)^{m+p} \mathrm{H}_{m}(\psi) \\
& \leq \frac{2^{m+p}}{\epsilon^{m+p}}(p+m) \ldots(p+1)\|\psi\|_{\infty}^{m} \int_{\Omega}\left(\varphi_{j}-u_{j}\right)^{p} \mathrm{H}_{m}\left(u_{j}\right) \\
& \leq \frac{2^{m+p}(p+m) \ldots(p+1)\|\psi\|_{\infty}^{m}}{\epsilon^{m+p}} \mathrm{~J}_{p}\left(u_{j}, \varphi_{j}\right)^{p+m} \rightarrow 0,
\end{aligned}
$$

as $j \rightarrow \infty$. 
Note that the reverse implications in Proposition 4.1 and Proposition 4.2 are not, in general, true. The following example is taken from [20].

\section{Example 4.3 Let}

$$
u_{j}(z)=\max \left(j^{\frac{p}{n}} \ln |z|,-\frac{1}{j}\right),
$$

be a plurisubharmonic function defined in the unit ball $\mathbb{B}$ in $\mathbb{C}^{n}, n \geq 2$. Then $u_{j} \in$ $\mathcal{E}_{0, n}(\mathbb{B})$,

$$
\mathrm{J}_{p}\left(u_{j}, 0\right)^{p+n}=e_{p, n}\left(u_{j}\right)=(2 \pi)^{n},
$$

but $u_{j} \rightarrow 0$ in capacity, and $u_{j} \rightarrow 0$ in $L^{p+n}(\mathbb{B})$.

At the end of this section, we shall prove that convergence in $\mathrm{J}_{p}$ implies weak convergence of the complex Hessian measures. We shall need the following lemma.

Lemma 4.4 Let $n \geq 2,1 \leq m \leq n$, and assume that $\Omega \subset \mathbb{C}^{n}$ is an m-hyperconvex domain and $u_{j}, u \in \mathcal{E}_{p, m}(\Omega)$ be such that $\sup _{j} e_{p, m}\left(u_{j}\right)<\infty$. Then, if $u_{j} \rightarrow u$ in capacity cap ${ }_{m}$, then $\mathrm{H}_{m}\left(u_{j}\right) \rightarrow \mathrm{H}_{m}(u)$ weakly, as $j \rightarrow \infty$.

\section{Proof Set}

$$
A=e_{p, m}(u)+\sup _{j} e_{p, m}\left(u_{j}\right)<\infty .
$$

Then for all $k, j$, we have

$$
e_{p, m}\left(\max \left(u_{j},-k\right)\right) \leq D(p, m)^{\frac{m+p}{p}} e_{p, m}\left(u_{j}\right) \leq D(m, p)^{\frac{m+p}{p}} A .
$$

Consider the following decomposition

$$
\begin{aligned}
\mathrm{H}_{m}\left(u_{j}\right)-\mathrm{H}_{m}(u)= & \left(\mathrm{H}_{m}\left(u_{j}\right)-\mathrm{H}_{m}\left(\max \left(u_{j},-k\right)\right)\right) \\
& +\left(\mathrm{H}_{m}\left(\max \left(u_{j},-k\right)\right)-\mathrm{H}_{m}(\max (u,-k))\right) \\
& +\left(\mathrm{H}_{m}(\max (u,-k))-\mathrm{H}_{m}(u)\right) \\
= & \mu_{j, k}^{1}+\mu_{j, k}^{2}+\mu_{k}^{3} .
\end{aligned}
$$

Furthermore, since $u_{j} \rightarrow u$ in capacity cap ${ }_{m}$, then for all $k$ we get $\max \left(u_{j},-k\right) \rightarrow$ $\max (u,-k)$, in capacity $\operatorname{cap}_{m}$. All functions are uniformly bounded, so by [30], $\mu_{j, k}^{2} \rightarrow 0$ weakly as $j \rightarrow \infty$. Since $\max (u,-k)$ is decreasing to $u$, as $k \rightarrow \infty$, and therefore $\mu_{k}^{3} \rightarrow 0$ weakly, as $k \rightarrow \infty$.

To finish the proof, we have to show that $\mu_{j, k}^{1} \rightarrow 0$, as $k \rightarrow \infty$ and uniformly on $j$. Let $\alpha \in \mathcal{C}_{0}^{\infty}(\Omega)$, and we shall use the temporary notation $T_{s}=$ $\left(d d^{c} \max \left(u_{j},-k\right)\right)^{m-s-1} \wedge \beta^{n-m}$. Then 


$$
\begin{aligned}
\left|\int_{\Omega} \alpha \mu_{j, k}^{1}\right|= & \left|\sum_{s=0}^{m-1} \int_{\Omega} \alpha\left(d d^{c} u_{j}\right)^{s} \wedge\left(d d^{c} u_{j}-d d^{c} \max \left(u_{j},-k\right)\right) \wedge T_{s}\right| \\
\leq & \|\alpha\|_{\infty} k^{-p} \sum_{s=0}^{m-1} \int_{\left\{u_{j} \leq-k\right\}}\left(-u_{j}\right)^{p}\left(d d^{c} u_{j}\right)^{s+1} \wedge T_{s} \\
& +\|\alpha\|_{\infty} k^{-p} \sum_{s=0}^{m-1} \int_{\left\{u_{j} \leq-k\right\}}\left(-u_{j}\right)^{p}\left(d d^{c} u_{j}\right)^{s} \wedge\left(d d^{c} \max \left(u_{j},-k\right)\right)^{m-s} \wedge \beta^{n-m} \\
\leq & \|\alpha\|_{\infty} k^{-p} \sum_{s=0}^{m-1} D(m, p) e_{p, m}\left(u_{j}\right)^{\frac{p+s+1}{p+m}} e_{p, m}\left(\max \left(u_{j},-k\right)\right)^{\frac{m-s-1}{p+m}} \\
& +\|\alpha\|_{\infty} k^{-p} \sum_{s=0}^{m-1} D(m, p) e_{p, m}\left(u_{j}\right)^{\frac{p+s}{p+m}} e_{p, m}\left(\max \left(u_{j},-k\right)\right)^{\frac{m-s}{p+m}} \\
\leq & \|\alpha\|_{\infty} k^{-p} 2 m D(m, p)^{\frac{m+p}{p}} A \rightarrow 0,
\end{aligned}
$$

as $k \rightarrow \infty$. The convergence above is uniform in $j$.

Proposition 4.5 Let $n \geq 2,1 \leq m \leq n$, and assume that $\Omega \subset \mathbb{C}^{n}$ is an $m$-hyperconvex domain. If $\mathrm{J}_{p}\left(u_{j}, u\right) \rightarrow 0$, as $j \rightarrow \infty$, then $\mathrm{H}_{m}\left(u_{j}\right) \rightarrow \mathrm{H}_{m}(u)$ weakly.

Proof Since

$$
e_{p, m}\left(u_{j}\right)^{\frac{1}{p+m}}=\mathbf{J}_{p}\left(u_{j}, 0\right) \leq C\left(\mathbf{J}_{p}\left(u_{j}, u\right)+\mathbf{J}_{p}(u, 0)\right),
$$

it follows

$$
e_{p, m}(u)+\sup _{j} e_{p, m}\left(u_{j}\right)<\infty
$$

This proof is then concluded by Proposition 4.2, and Lemma 4.4.

\section{A Comparison of Different Topologies}

In this section, we begin by comparing the quasimetric space $\left(\mathcal{E}_{1, m}(\Omega), \mathrm{J}_{1}\right)$ with the metric space $\left(\mathcal{E}_{1, m}(\Omega)\right.$, d) studied in [8]. In the second part of this section, we show that the topology generated by $\mathrm{J}_{p}$ is not comparable with the topology generated by the subspace $\mathcal{E}_{p, m}(\Omega)$ of quasi-normed space $\left(\delta \mathcal{E}_{p, m}(\Omega),\|\cdot\|_{p}\right)$ studied in [33]. The presentation of the latter part follows closely Sect. 7 of [8].

Let us first introduce the necessary definitions and notations and formulate the results that are relevant here. For further information, see [8].

Definition 5.1 Let $1 \leq m \leq n$, and let $\Omega$ be a bounded $m$-hyperconvex domain in $\mathbb{C}^{n}, n>1$. Fix $w \in \mathcal{E}_{1, m}(\Omega)$, known as the weight, and define the weighted energy functional $\mathrm{E}_{w}$ by 


$$
\mathcal{E}_{1, m}(\Omega) \ni u \mapsto \mathrm{E}_{w}(u)=\frac{1}{m+1} \sum_{j=0}^{m} \int_{\Omega}(u-w)\left(d d^{c} u\right)^{j} \wedge\left(d d^{c} w\right)^{m-j} \wedge \beta^{n-m} \in \mathbb{R}
$$

Definition 5.2 Let $n \geq 2,1 \leq m \leq n$, and assume that $\Omega \subset \mathbb{C}^{n}$ is an $m$-hyperconvex domain. Fix $w, u, v \in \mathcal{E}_{1, m}(\Omega)$. Let us define

$$
\mathbf{d}(u, v)=\mathrm{E}_{w}(u)+\mathrm{E}_{w}(v)-2 \mathrm{E}_{w}(\mathrm{P}(u, v))
$$

Theorem 5.3 Let $n \geq 2,1 \leq m \leq n$, and let $\Omega$ be a bounded $m$-hyperconvex domain in $\mathbb{C}^{n}$. Then the tuple $\left(\mathcal{E}_{1, m}(\Omega), \overline{\mathbf{d}}\right)$ is a complete metric space.

Proof See Theorem 5.3 in [8].

Now we are in position to compare the metric $\mathbf{d}$ with the quasimetric $\mathrm{J}_{1}$.

Theorem 5.4 Let $n \geq 2,1 \leq m \leq n$, and assume that $\Omega \subset \mathbb{C}^{n}$ is an m-hyperconvex domain. Then for $u, v \in \mathcal{E}_{1, m}(\Omega)$ it holds

$$
\frac{1}{2^{m+2} C^{m+1}(m+1)} \mathbf{J}_{1}(u, v)^{m+1} \leq \mathbf{d}(u, v) \leq \mathbf{J}_{1}(u, v)^{m+1},
$$

where $C$ is a constant from quasi-triangle inequality.

Proof Let $u, v \in \mathcal{E}_{1, m}(\Omega)$. Note that $\{\mathrm{P}(u, v)=u\} \subset\{u \leq v\}$ and $\{\mathrm{P}(u, v)=v\} \subset$ $\{v \leq u\}$. Then we obtain by Theorem 3.3 and [8, Proposition 3.3]

$$
\begin{aligned}
& \mathbf{d}(u, v)=\mathrm{E}_{w}(u)-\mathrm{E}_{w}(\mathrm{P}(u, v))+\mathrm{E}_{w}(v)-\mathrm{E}_{w}(\mathrm{P}(u, v)) \\
& \leq \int_{\Omega}(u-\mathrm{P}(u, v)) \mathrm{H}_{m}(\mathrm{P}(u, v))+\int_{\Omega}(v-\mathrm{P}(u, v)) \mathrm{H}_{m}(\mathrm{P}(u, v)) \\
& =\int_{\{\mathrm{P}(u, v)<u\}}(u-\mathrm{P}(u, v))\left(\chi_{\{\mathrm{P}(u, v)=v\}} \mathrm{H}_{m}(v)+\chi_{\{\mathrm{P}(u, v)=u\}} \mathrm{H}_{m}(u)\right) \\
& +\int_{\{\mathrm{P}(u, v)<v\}}(v-\mathrm{P}(u, v))\left(\chi_{\{\mathrm{P}(u, v)=v\}} \mathrm{H}_{m}(v)+\chi_{\{\mathrm{P}(u, v)=u\}} \mathrm{H}_{m}(u)\right) \\
& \leq \int_{\{\mathrm{P}(u, v)<u\} \cap\{\mathrm{P}(u, v)=v\}}(u-\mathrm{P}(u, v)) \mathrm{H}_{m}(v) \\
& +\int_{\{\mathrm{P}(u, v)<v\} \cap\{\mathrm{P}(u, v)=u\}}(v-\mathrm{P}(u, v)) \mathrm{H}_{m}(u) \\
& \leq \int_{\{v<u\}}(u-v) \mathrm{H}_{m}(v)+\int_{\{u<v\}}(v-u) \mathrm{H}_{m}(u) \\
& \leq \int_{\{v<u\}}(u-v)\left(\mathrm{H}_{m}(v)+\mathrm{H}_{m}(u)\right)+\int_{\{u<v\}}(v-u)\left(\mathrm{H}_{m}(u)+\mathrm{H}_{m}(v)\right) \\
& =\mathrm{J}_{1}(u, v)^{m+1} \text {. }
\end{aligned}
$$


Thanks to Proposition 3.4 (6), the quasi-triangle inequality, and [8, Proposition 3.3] we get

$$
\begin{aligned}
\mathbf{d}(u, v) & =\mathrm{E}_{w}(u)-\mathrm{E}_{w}(\mathrm{P}(u, v))+\mathrm{E}_{w}(v)-\mathrm{E}_{w}(\mathrm{P}(u, v)) \\
& \geq \frac{1}{m+1} \int_{\Omega}(u-\mathrm{P}(u, v)) \mathrm{H}_{m}(\mathrm{P}(u, v))+\frac{1}{m+1} \int_{\Omega}(v-\mathrm{P}(u, v)) \mathrm{H}_{m}(\mathrm{P}(u, v)) \\
& \geq \frac{1}{2(m+1)} \mathrm{J}_{1}(u, \mathrm{P}(u, v))^{m+1}+\frac{1}{2(m+1)} \mathrm{J}_{1}(v, \mathrm{P}(u, v))^{m+1} \\
& \geq \frac{1}{2^{m+2}(m+1)}\left(\mathrm{J}_{1}(u, \mathrm{P}(u, v))+\mathrm{J}_{1}(v, \mathrm{P}(u, v))\right)^{m+1} \\
& \geq \frac{1}{2^{m+2} C^{m+1}(m+1)} \mathrm{J}_{1}(u, v)^{m+1}
\end{aligned}
$$

Now to the second part of this section. Let us start here with a brief introduction. We start by defining

$$
\delta \mathcal{E}_{p, m}(\Omega)=\mathcal{E}_{p, m}(\Omega)-\mathcal{E}_{p, m}(\Omega)
$$

since $\mathcal{E}_{p, m}(\Omega)$ is only a convex cone. Then for any $u \in \delta \mathcal{E}_{p, m}(\Omega)$ we define

$$
\|u\|_{p}=\inf _{\substack{u_{1}-u_{2}=u \\ u_{1}, u_{2} \in \mathcal{E}_{p, m}(\Omega)}}\left(\int_{\Omega}\left(-\left(u_{1}+u_{2}\right)\right)^{p} \mathrm{H}_{m}\left(u_{1}+u_{2}\right)\right)^{\frac{1}{m+p}} .
$$

It was proved in [33] that $\left(\delta \mathcal{E}_{p, m}(\Omega),\|\cdot\|_{p}\right)$ is a quasi-Banach space, i.e., it is complete quasi-normed vector space (for the case $m=n$ see [4]). Recall that $\|\cdot\|_{p}$ is a quasinorm if the following holds:

(1) $\|u\|_{p}=0$ if, and only if, $u=0$;

(2) $\|t u\|_{p}=|t|\|u\|_{p}$;

(3) it satisfies quasi-triangle inequality

$$
\|u+v\|_{p} \leq C\left(\|u\|_{p}+\|v\|_{p}\right)
$$

for some constant $C \geq 1$.

Furthermore, if $u \in \mathcal{E}_{p, m}(\Omega)$, then $\|u\|_{p}=e_{p, m}(u)^{\frac{1}{p+m}}$.

Example 5.5 There is no constant $C>0$ such that $\mathrm{J}_{p}(u, v) \leq C\|u-v\|_{p}$. See Example 7.1 in [8].

Example 5.6 There is no constant $C>0$ such that $\|u-v\|_{p} \leq C \mathrm{~J}_{p}(u, v)$. See Example 7.2 in [8]. 


\section{Stability of the Complex Hessian Operator}

Let

$$
\begin{gathered}
\mathcal{M}_{p, m}=\{\mu: \mu \text { is a non-negative Radon measure on } \Omega \text { such that } \\
\left.\mathrm{H}_{m}(u)=\mu \text { for some } u \in \mathcal{E}_{p, m}\right\},
\end{gathered}
$$

and recall the following characterization of $\mathcal{M}_{p, m}$ :

(1) $\mu \in \mathcal{M}_{p, m}$;

(2) there exists a constant $A \geq 0$ such that

$$
\int_{\Omega}(-u)^{p} d \mu \leq A e_{p, m}(u)^{\frac{p}{p+m}} \quad \text { for all } u \in \mathcal{E}_{p, m}(\Omega)
$$

(3) $\mathcal{E}_{p, m}(\Omega) \subset L^{p}(\mu)$;

(4) there exists unique function $U(\mu) \in \mathcal{E}_{p, m}(\Omega)$ the solution to the Dirichlet problem for the complex Hessian equation $\mathrm{H}_{m}(U(\mu))=\mu$,

(see $[15,30]$ for details).

In this section, we shall prove some new stability results for the complex Hessian operator. For previous results concerning stability of the complex Monge-Ampère equation or the complex Hessian equation, see, e.g., [16,19,34]. In those papers, the authors proved that under some assumption if $\mu_{j}$ converges to $\mu$, then the corresponding solutions $U\left(\mu_{j}\right)$ converges to $U(\mu)$ in capacity. Our goal is to prove that the convergence is stronger in the sense that $\mathrm{J}_{p}\left(U\left(\mu_{j}\right), U(\mu)\right) \rightarrow 0$.

Lemma 6.1 Let $n \geq 2,1 \leq m \leq n$, and assume that $\Omega \subset \mathbb{C}^{n}$ is an m-hyperconvex domain. Let $\mu \in \mathcal{M}_{p, m}$. Then for any $v \leq \mu$ it holds:

$$
e_{p, m}(U(v)) \leq D(p, m)^{\frac{m+p}{p}} e_{p, m}(U(\mu)) .
$$

Proof The desired inequality follows from the following estimation

$$
\begin{aligned}
e_{p, m}(U(v)) & =\int_{\Omega}(-U(v))^{p} \mathrm{H}_{m}(U(v))=\int_{\Omega}(-U(v))^{p} \mathrm{~d} v \leq \int_{\Omega}(-U(v))^{p} \mathrm{~d} \mu \\
& =\int_{\Omega}(-U(v))^{p} \mathrm{H}_{m}(U(\mu)) \leq D(m, p) e_{p, m}(U(v))^{\frac{p}{p+m}} e_{p, m}(U(\mu))^{\frac{m}{m+p}} .
\end{aligned}
$$

Definition 6.2 A fundamental sequence $\Omega_{j}, j \in \mathbb{N}$, is an increasing sequence of $m$ hyperconvex subsets of $\Omega \subset \mathbb{C}^{n}, n \geq 2$, such that for every $j \in \mathbb{N}$, we have that, $\Omega_{j} \Subset \Omega_{j+1}$, and $\bigcup_{j=1}^{\infty} \Omega_{j}=\Omega$.

The main result in this section is the following stability theorem. 
Theorem 6.3 Let $n \geq 2,1 \leq m \leq n$, and assume that $\Omega \subset \mathbb{C}^{n}$ is an m-hyperconvex domain and let $\mu \in \mathcal{M}_{p, m}$. If $0 \leq f, f_{j} \leq 1$ are measurable functions such that $f_{j} \rightarrow f$ in $L_{\text {loc }}^{1}(\mu)$, as $j \rightarrow \infty$, then $\mathrm{J}_{p}\left(U\left(f_{j} \mu\right), U(f \mu)\right) \rightarrow 0$.

Proof Fix $\mu \in \mathcal{M}_{p, m}$. From the Cegrell-Lebesgue decomposition theorem (see [15, 30]), it follows that there exist $\varphi \in \mathcal{E}_{0, m}(\Omega),\|\varphi\|_{\infty} \leq 1$, and $g \geq 0$ such that $g \mathrm{H}_{m}(\varphi)=\mu$. Fix a fundamental sequence $\Omega_{j}$. For $j, k \in \mathbb{N}$, let us define the following functions:

$$
\begin{array}{ll}
w=U(\mu) \in \mathcal{E}_{p, m}(\Omega): & \mathrm{H}_{m}(w)=\mu ; \\
u=U(f \mu) \in \mathcal{E}_{p, m}(\Omega): & \mathrm{H}_{m}(u)=f \mu ; \\
u_{j}=U\left(f_{j} \mu\right) \in \mathcal{E}_{p, m}(\Omega): & \mathrm{H}_{m}\left(u_{j}\right)=f_{j} \mu ; \\
u_{j, k} \in \mathcal{E}_{p, m}(\Omega): & \mathrm{H}_{m}\left(u_{j, k}\right)=\chi_{\Omega_{k}} f_{j} \min (g, k) \mathrm{H}_{m}(\varphi) ; \\
v_{j, k} \in \mathcal{E}_{p, m}(\Omega): & \mathrm{H}_{m}\left(v_{j, k}\right)=\chi_{\Omega_{k}} f_{j} \mu ; \\
w_{k} \in \mathcal{E}_{p, m}(\Omega): & \mathrm{H}_{m}\left(w_{k}\right)=\chi_{\Omega_{k}} f \min (g, k) \mathrm{H}_{m}(\varphi) ; \\
v_{k} \in \mathcal{E}_{p, m}(\Omega): & \mathrm{H}_{m}\left(v_{k}\right)=\left(1-\chi_{\Omega_{k}}\right) \mu ; \\
\psi_{k} \in \mathcal{E}_{p, m}(\Omega): & \mathrm{H}_{m}\left(\psi_{k}\right)=(g-\min (g, k)) \mathrm{H}_{m}(\varphi)
\end{array}
$$

Since,

$$
\mathbf{J}_{p}\left(u, u_{j}\right) \leq C^{3}\left(\mathbf{J}_{p}\left(u, w_{k}\right)+\mathbf{J}_{p}\left(w_{k}, u_{j, k}\right)+\mathbf{J}_{p}\left(u_{j, k}, v_{j, k}\right)+\mathbf{J}_{p}\left(v_{j, k}, u_{j}\right)\right),
$$

it is enough to prove that each term in (6.1) tends to zero to complete the proof.

Claim 1. $\mathbf{J}_{p}\left(u, w_{k}\right) \rightarrow 0$, as $k \rightarrow \infty$. This follows because $w_{k}$ is a decreasing sequence tending to $u$, as $k \rightarrow \infty$.

Claim 2. For fixed $k$, we have that $\mathrm{J}_{p}\left(w_{k}, u_{j, k}\right) \rightarrow 0$, as $j \rightarrow \infty$. To prove this, first note that the functions $w_{k}$ and $u_{j, k}$ are uniformly bounded by $k^{\frac{1}{m}}$, and therefore, it follows

$$
\begin{aligned}
\mathrm{J}_{p}\left(w_{k}, u_{j, k}\right)^{p+m}= & \int_{\Omega}\left|w_{k}-u_{j, k}\right|^{p}\left(\mathrm{H}_{m}\left(w_{k}\right)+\mathrm{H}_{m}\left(u_{j, k}\right)\right) \\
= & \int_{\left\{w_{k}<u_{j, k}\right\}}\left(u_{j, k}-w_{k}\right)^{p} \chi_{\Omega_{k}}\left(f_{j}+f\right) \min (g, k) \mathrm{H}_{m}(\varphi) \\
& +\int_{\left\{w_{k}>u_{j, k}\right\}}\left(w_{k}-u_{j, k}\right)^{p} \chi_{\Omega_{k}}\left(f_{j}+f\right) \min (g, k) \mathrm{H}_{m}(\varphi) \\
\leq & 2 k \int_{\left\{w_{k}<u_{j, k}\right\}}\left(u_{j, k}-w_{k}\right)^{p} \mathrm{H}_{m}(\varphi)+2 k \int_{\left\{w_{k}>u_{j, k}\right\}}\left(w_{k}-u_{j, k}\right)^{p} \mathrm{H}_{m}(\varphi) .
\end{aligned}
$$

Now assume that $p \leq m$. Then we can continue our estimate by using the Hölder inequality. By [34], we get 


$$
\begin{aligned}
& \int_{\left\{w_{k}<u_{j, k}\right\}}\left(u_{j, k}-w_{k}\right)^{p} \mathrm{H}_{m}(\varphi) \\
& \leq\left(\int_{\left\{w_{k}<u_{j, k}\right\}}\left(u_{j, k}-w_{k}\right)^{m} \mathrm{H}_{m}(\varphi)\right)^{\frac{p}{m}}\left(\mathrm{H}_{m}(\varphi)(\Omega)\right)^{\frac{m-p}{m}} \\
& \leq\left(\mathrm{H}_{m}(\varphi)(\Omega)\right)^{\frac{m-p}{m}}\left(m ! \int_{\left\{w_{k}<u_{j, k}\right\}}(-\varphi)\left(\mathrm{H}_{m}\left(w_{k}\right)-\mathrm{H}_{m}\left(u_{j, k}\right)\right)\right)^{\frac{p}{m}} \\
& \leq\left(\mathrm{H}_{m}(\varphi)(\Omega)\right)^{\frac{m-p}{m}}\left(m ! \int_{\Omega_{k}}\left|f_{j}-f\right| d \mu\right)^{\frac{p}{m}} \rightarrow 0, \text { as } j \rightarrow \infty .
\end{aligned}
$$

In a similar way, one can prove

$$
\int_{\left\{w_{k}>u_{j, k}\right\}}\left(w_{k}-u_{j, k}\right)^{p} \mathrm{H}_{m}(\varphi) \leq\left(\mathrm{H}_{m}(\varphi)(\Omega)\right)^{\frac{m-p}{m}}\left(m ! \int_{\Omega_{k}}\left|f_{j}-f\right| d \mu\right)^{\frac{p}{m}} \rightarrow 0,
$$

as $j \rightarrow \infty$. If $p>m$, then one can repeat the above argument using the fact

$$
\left|u_{j, k}-w_{k}\right|^{p} \leq\left(2 k^{\frac{1}{m}}\right)^{p-m}\left|u_{j, k}-w_{k}\right|^{m} .
$$

Claim 3. $\mathrm{J}_{p}\left(v_{j, k}, u_{j, k}\right) \rightarrow 0$, as $k \rightarrow \infty$, and the convergence is uniform on $j$. Since

$$
\begin{aligned}
\mathrm{H}_{m}\left(v_{j, k}\right) & =\chi_{\Omega_{k}} f_{j} g \mathrm{H}_{m}(\varphi) \\
& \leq \chi \Omega_{k} f_{j} \min (g, k) \mathrm{H}_{m}(\varphi)+(g-\min (g, k)) \mathrm{H}_{m}(\varphi) \\
& =\mathrm{H}_{m}\left(u_{j, k}\right)+\mathrm{H}_{m}\left(\psi_{k}\right) \leq \mathrm{H}_{m}\left(u_{j, k}+\psi_{k}\right),
\end{aligned}
$$

we have that $u_{j, k}+\psi_{k} \leq v_{j, k}$. Furthermore, $u_{j, k} \geq v_{j, k}$, and $\psi_{k}$ is a increasing sequence such that

$$
\begin{aligned}
e_{p, m}\left(\psi_{k}\right) & =\int_{\Omega}\left(-\psi_{k}\right)^{p} \mathrm{H}_{m}\left(\psi_{k}\right)=\int_{\Omega}\left(-\psi_{k}\right)^{p}(g-\min (g, k)) \mathrm{H}_{m}(\varphi) \\
& \leq \int_{\Omega}\left(-\psi_{1}\right)^{p}(g-\min (g, k)) \mathrm{H}_{m}(\varphi) \rightarrow 0, k \rightarrow \infty,
\end{aligned}
$$

by dominated convergence theorem. Finally,

$$
\begin{aligned}
\mathrm{J}_{p}\left(v_{j, k}, u_{j, k}\right)^{p+m} & =\int_{\Omega}\left|v_{j, k}-u_{j, k}\right|^{p}\left(\mathrm{H}_{m}\left(v_{j, k}\right)+\mathrm{H}_{m}\left(u_{j, k}\right)\right) \\
& \leq 2 \int_{\Omega}\left(u_{j, k}-v_{j, k}\right)^{p} \mathrm{H}_{m}(w) \leq 2 \int_{\Omega}\left(-\psi_{k}\right)^{p} \mathrm{H}_{m}(w) \\
& \leq 2 D(m, p) e_{p, m}\left(\psi_{k}\right)^{\frac{p}{m+p}} e_{p, m}(w)^{\frac{m}{m+p}} \rightarrow 0
\end{aligned}
$$

as $k \rightarrow \infty$. The convergence is as well uniform in $j$. 
Claim 4. $\mathrm{J}_{p}\left(v_{j, k}, u_{j}\right) \rightarrow 0$, as $k \rightarrow \infty$, and the convergence is uniform on $j$. The proof of Claim 4 follows that of Claim 3. Observe that

$$
\begin{aligned}
\mathrm{H}_{m}\left(u_{j}\right) & =f_{j} \mu \leq\left(1-\chi \Omega_{k}\right) \mu+f_{j} \chi \Omega_{k} \mu \\
& =\mathrm{H}_{m}\left(v_{k}\right)+\mathrm{H}_{m}\left(v_{j, k}\right) \leq \mathrm{H}_{m}\left(v_{k}+v_{j, k}\right),
\end{aligned}
$$

which implies that $v_{k}+v_{j, k} \leq u_{j}$. Furthermore, $u_{j} \leq v_{j, k}$, and $v_{k}$ is increasing sequence such that

$$
\begin{aligned}
e_{p, m}\left(v_{k}\right) & =\int_{\Omega}\left(-v_{k}\right)^{p} \mathrm{H}_{m}\left(v_{k}\right)=\int_{\Omega}\left(-v_{k}\right)^{p}\left(1-\chi_{\Omega_{k}}\right) \mu \\
& \leq \int_{\Omega}\left(-v_{1}\right)^{p}\left(1-\chi_{\Omega_{k}}\right) \mu \rightarrow 0, k \rightarrow \infty,
\end{aligned}
$$

by dominated convergence theorem. Finally,

$$
\begin{aligned}
\mathrm{J}_{p}\left(v_{j, k}, u_{j}\right)^{p+m} & =\int_{\Omega}\left|v_{j, k}-u_{j}\right|^{p}\left(\mathrm{H}_{m}\left(v_{j, k}\right)+\mathrm{H}_{m}\left(u_{j}\right)\right) \\
& \leq 2 \int_{\Omega}\left(v_{j, k}-u_{j}\right)^{p} \mathrm{H}_{m}(w) \leq 2 \int_{\Omega}\left(-v_{k}\right)^{p} \mathrm{H}_{m}(w) \\
& \leq 2 D(m, p) e_{p, m}\left(v_{k}\right)^{\frac{p}{m+p}} e_{p, m}(w)^{\frac{m}{m+p}} \rightarrow 0, k \rightarrow \infty,
\end{aligned}
$$

the convergence is uniform in $j$.

\section{The Compact Kähler Manifold Case}

Let $n \geq 2, p>0$, and let $1 \leq m \leq n$. Assume that $(X, \omega)$ is a connected and compact Kähler manifold of complex dimension $n$, where $\omega$ is a Kähler form on $X$ such that $\int_{X} \omega^{n}=1$. In a similar way as in Sect. 3 , we define a quasimetric, $I_{p}$, for $(\omega, m)$-subharmonic functions (for the case $m=n$, see [25]). For further information concerning $(\omega, m)$-subharmonic function $\left(\mathcal{S H}_{m}(X, \omega)\right)$ on compact Kähler manifold, see, e.g., [7,21,25,31].

For any $u \in \mathcal{S H}_{m}(X, \omega)$, let

$$
\omega_{u}=d d^{c} u+\omega
$$

The complex Hessian operator is defined on $(\omega, m)$-subharmonic functions through the following construction: First assume that $u \in \mathcal{S H}_{m}(X, \omega) \cap L^{\infty}(X)$, then

$$
\mathrm{H}_{m}(u):=\omega_{u}^{m} \wedge \omega^{n-m}
$$

which is a non-negative (regular) Borel measure defined on $X$. For an arbitrary, not necessarily bounded, $(\omega, m)$-subharmonic function $u$ let $u_{j}=\max (u,-j)$ be the 
canonical approximation of $u$. Then define

$$
\mathrm{H}_{m}(u):=\lim _{j \rightarrow \infty} \chi\{u>-j\} \mathrm{H}_{m}\left(u_{j}\right)
$$

We define the class of $(\omega, m)$-subharmonic functions with bounded $(p, m)$-energy as

$$
\mathcal{E}_{p, m}(X, \omega):=\left\{u \in \mathcal{E}_{m}(X, \omega): u \leq 0, \int_{X}(-u)^{p} \mathrm{H}_{m}(u)<\infty\right\}
$$

where

$$
\mathcal{E}_{m}(X, \omega)=\left\{u \in \mathcal{S} \mathcal{H}_{m}(X, \omega): \int_{X} \mathrm{H}_{m}(u)=1\right\}
$$

For the counterparts of Theorems 2.1, 2.2, and the approximation theorem for $(\omega, m)$ subharmonic functions defined on compact Kähler manifolds, we refer to [7,21,25,31, 32].

The following definition is the counterpart of $\mathrm{J}_{p}$ in Definition 3.1.

Definition 7.1 Let $n \geq 2, p>0$, and let $1 \leq m \leq n$. For $u, v \in \mathcal{E}_{p, m}(X, \omega)$ and we define

$$
\mathrm{I}_{p}(u, v)=\left(\int_{X}|u-v|^{p}\left(\mathrm{H}_{m}(u)+\mathrm{H}_{m}(v)\right)\right)^{\frac{1}{p+m}} .
$$

Remark Note that it follows from [7, Lemma 3.5] that the functional $\mathrm{I}_{p}$ is well defined.

The aim of this section is to prove that $\left(\mathcal{E}_{p, m}(X, \omega), \mathrm{I}_{p}\right)$ is a complete quasimetric space.

Theorem 7.2 The pair $\left(\mathcal{E}_{p, m}(X, \omega), \mathrm{I}_{p}\right)$ is a quasimetric space.

Proof First assume that $u, v \in \mathcal{E}_{p, m}(X, \omega)$, and $\mathrm{I}_{p}(u, v)=0$. Then $\mathrm{H}_{m}(u)(\{u<$ $v\})=0$, and by [31, Theorem 3.15] we get $u \geq v$. In a similar way, we can obtain that $v \geq u$. Hence, $u=v$. The quasi-triangle inequality follows in the same way as in Lemma 3.5.

We shall need the counterpart of Proposition 2.4.

Proposition 7.3 Let $u, v \in \mathcal{E}_{p, m}(X, \omega)$.

(1) If $u \leq v$, then

$$
\int_{X}(v-u)^{p} \mathrm{H}_{m}(v) \leq \int_{X}(v-u)^{p} \mathrm{H}_{m}(u) .
$$

(2) Without any additional assumption on $u$, and $v$, it holds

$$
\int_{\{u<v\}}(v-u)^{p} \mathrm{H}_{m}(v) \leq \int_{\{u<v\}}(v-u)^{p} \mathrm{H}_{m}(u) .
$$


Proof (1) First assume that $u<v$. Then for any positive current $T$ it holds

$$
\begin{aligned}
& \int_{X}(v-u)^{p} \omega_{u} \wedge T-\int_{X}(v-u)^{p} \omega_{v} \wedge T \\
& \quad=p \int_{X}(v-u)^{p-1} d(v-u) \wedge d^{c}(v-u) \wedge T \geq 0 .
\end{aligned}
$$

Now, let $\epsilon<1$, then $u \leq v<\epsilon v$. We obtain

$$
\epsilon \int_{X}(\epsilon v-u)^{p} \omega_{v} \wedge T \leq \int_{X}(\epsilon v-u)^{p} \omega_{\epsilon v} \wedge T \leq \int_{X}(\epsilon v-u)^{p} \omega_{u} \wedge T,
$$

so by the monotone convergence theorem, and letting $\epsilon \rightarrow 1^{-}$, we arrive ay

$$
\int_{X}(v-u)^{p} \omega_{v} \wedge T \leq \int_{X}(v-u)^{p} \omega_{v} \wedge T
$$

Repeating the above argument $m$-times we get

$$
\int_{X}(v-u)^{p} \mathrm{H}_{m}(v) \leq \int_{X}(v-u)^{p} \mathrm{H}_{m}(u) .
$$

(2) This part follows now in a similar manner as in Proposition 2.4.

Corollary 7.4 Let $u, u_{j}, v \in \mathcal{E}_{p, m}(X, \omega)$.

(1) If $u \leq v$, then

$$
2 \int_{X}(u-v)^{p} \mathrm{H}_{m}(v) \leq \mathrm{I}_{p}(u, v)^{p+m} \leq 2 \int_{X}(u-v)^{p} \mathrm{H}_{m}(u) .
$$

(2) If $u_{j} \searrow u, j \rightarrow \infty$, then $\mathrm{I}_{p}\left(u_{j}, u\right) \rightarrow 0$.

We end this paper with the main result of the compact Kähler case.

Theorem 7.5 The pair $\left(\mathcal{E}_{p, m}(X, \omega), \mathrm{I}_{p}\right)$ is a complete quasimetric space.

Proof Theorem 7.2 gives that $\left(\mathcal{E}_{p, m}(X, \omega), \mathrm{I}_{p}\right)$ is a quasimetric space, and the completeness can be proved in exactly the same way as in Theorem 3.9.

Funding Open access funding provided by Umeå University.

Open Access This article is licensed under a Creative Commons Attribution 4.0 International License, which permits use, sharing, adaptation, distribution and reproduction in any medium or format, as long as you give appropriate credit to the original author(s) and the source, provide a link to the Creative Commons licence, and indicate if changes were made. The images or other third party material in this article are included in the article's Creative Commons licence, unless indicated otherwise in a credit line to the material. If material is not included in the article's Creative Commons licence and your intended use is not permitted by statutory regulation or exceeds the permitted use, you will need to obtain permission directly from the copyright holder. To view a copy of this licence, visit http://creativecommons.org/licenses/by/4.0/. 


\section{References}

1. Abdullaev, B.I., Sadullaev, A.: Potential theory in the class of $m$-subharmonic functions. Proc. Steklov Inst. Math. 279(1), 155-180 (2012)

2. Abdullaev, B.I., Sadullaev, A.: Capacities and Hessians in the class of $m$-subharmonic functions. Dokl. Math. 87(1), 88-90 (2013)

3. Åhag, P., Czyż, R., Phạm, H.H.: Concerning the energy class $\mathcal{E}$ for $0<p<1$. Ann. Polon. Math. 91(2-3), 119-130 (2007)

4. Åhag, P., Czyż, R.: Modulability and duality of certain cones in pluripotential theory. J. Math. Anal. Appl. 361(2), 302-321 (2010)

5. Åhag, P., Czyż, R., Hed, L.: The geometry of $m$-hyperconvex domains. J. Geom. Anal. 28(4), 3196$3222(2018)$

6. Åhag, P., Czyż, R.: Poincaré-and Sobolev-type inequalities for complex $m$-Hessian equations. Results Math. 75(2), Paper No. 63, 21 pp (2020)

7. Åhag, P., Czyż, R.: A characterization of the degenerate complex Hessian equations for functions with bounded ( $p, m)$-energy. Manuscript (2020), arXiv:2003.06157

8. Åhag, P., Czyż, R.: Geodesics in the space of $m$-subharmonic functions with bounded energy. Manuscript (2021), arXiv:2110.02604

9. Bedford, E., Taylor, B.A.: Variational properties of the complex Monge-Ampère equation. I. Dirichlet principle. Duke Math. J. 45(2), 375-403 (1978)

10. Bedford, E., Taylor, B.A.: Variational properties of the complex Monge-Ampère equation. II. Intrinsic norms. Am. J. Math. 101(5), 1131-1166 (1979)

11. Berman, J., Boucksom, S., Eyssidieux, P., Guedj, V., Zeriahi, A.: Kähler-Einstein metrics and the Kähler-Ricci flow on log Fano varieties. J. Reine Angew. Math. 751, 27-89 (2019)

12. Błocki, Z.: Weak solutions to the complex Hessian equation. Ann. Inst. Fourier (Grenoble) 55(5), 1735-1756 (2005)

13. Calabuig, J.M., Falciani, H., Sánchez-Pérez, E.A.: Dreaming machine learning: Lipschitz extensions for reinforcement learning on financial markets. Neurocomputing 398, 172-184 (2020)

14. Caffarelli, L., Nirenberg, L., Spruck, J.: The Dirichlet problem for nonlinear second-order elliptic equations. III. Functions of the eigenvalues of the Hessian. Acta Math. 155(3-4), 261-301 (1985)

15. Cegrell, U.: Pluricomplex energy. Acta Math. 180(2), 187-217 (1998)

16. Cegrell, U., Kołodziej, S.: The equation of complex Monge-Ampère type and stability of solutions. Math. Ann. 334(4), 713-729 (2006)

17. Cegrell, U., Persson, L.: An energy estimate for the complex Monge-Ampère operator. Ann. Polon. Math. 67(1), 95-102 (1997)

18. Chern, S.S., Levine, H.I., Nirenberg, L.: Intrinsic norms on a complex manifold. 1969 Global Analysis (Papers in Honor of K. Kodaira), pp. 119-139, Univ. Tokyo Press, Tokyo

19. Czyż, R.: The complex Monge-Ampère operator in the Cegrell classes. Dissertationes Math. 466, 83 (2009)

20. Czyż, R.: A note on Le-Pham's paper-convergence in $\mathcal{E}_{p}$ spaces. Acta Math. Vietnam. 34(3), 401-410 (2009)

21. Dinew, S., Lu, H.-C.: Mixed Hessian inequalities and uniqueness in the class $\mathcal{E}(X, \omega, m)$. Math. Z. 279(3-4), 753-766 (2015)

22. Di Nezza, E., Lu, C.-H.: $L^{p}$ metric geometry of big and nef cohomology classes. Acta Math. Vietnam. 45(1), 53-69 (2020)

23. Gaveau, B.: Méthodes de contrôle optimal en analyse complexe; résolution d'équations de MongeAmpère. C. R. Acad. Sci. Paris Sér. A 284(11), A593-A596 (1977)

24. Gaveau, B.: Méthodes de contrôle optimal en analyse complexe. I. Résolution d'équations de MongeAmpère. J. Funct. Anal. 25(4), 391-411 (1977)

25. Guedj, V., Lu, H.-C., Zeriahi, A.: Plurisubharmonic envelopes and supersolutions. J. Differ. Geom. 113(2), 273-313 (2019)

26. Heinonen, J.: Lectures on Analysis on Metric Spaces. Universitext. Springer, New York (2001)

27. Jäkel, F., Schölkopf, B., Wichmann, F.A.: Similarity, kernels, and the triangle inequality. J. Math. Psych. 52(5), 297-303 (2008)

28. Kalina, J.: Some remarks on variational properties of inhomogeneous complex Monge-Ampère equation. Bull. Polish Acad. Sci. Math. 31(1-2), 9-14 (1983)

29. Lu, H.-C.: Complex Hessian equations. Doctoral thesis. University of Toulouse III Paul Sabatier (2012) 
30. Lu, H.-C.: A variational approach to complex Hessian equations in $\mathbb{C}^{n}$. J. Math. Anal. Appl. 431(1), 228-259 (2015)

31. Lu, H.-C., Nguyê̂n, V.-D.: Degenerate complex Hessian equations on compact Kähler manifolds. Indiana Univ. Math. J. 64(6), 1721-1745 (2015)

32. Lu, H.-C., Nguyê̂n, V.-D.: Complex Hessian equations with prescribed singularity on compact Kähler manifolds. Ann. Sc. Norm. Super. Pisa Cl. Sci. (accepted). Manuscript (2019), arXiv:1909.02469

33. Nguyễn, V.-T.: On delta $m$-subharmonic functions. Ann. Polon. Math. 118(1), 25-49 (2016)

34. Nguyễn, V.-T.: ., Maximal $m$-subharmonic functions and the Cegrell class $\mathcal{N}_{m}$. Indag. Math. (N.S.) 30(4), 717-739 (2019)

35. Persson, L.: A Dirichlet principle for the complex Monge-Ampère operator. Ark. Mat. 37(2), 345-356 (1999)

36. Shepard, R.N.: Attention and the metric structure of the stimulus space. J. Math. Psych. 1(1), 54-87 (1964)

37. Xing, Y.: A strong comparison principle for plurisubharmonic functions with finite pluricomplex energy. Michigan Math. J. 56(3), 563-581 (2008)

38. Vinacua, A.: Nonlinear elliptic equations written in terms of functions of the eigenvalues of the complex Hessian. Doctoral thesis, New York University, (1986)

39. Vinacua, A.: Nonlinear elliptic equations and the complex Hessian. Commun. Partial Differ. Eqs. 13(12), 1467-1497 (1988)

40. Zolotarev, V.M.: Metric distances in spaces of random variables and of their distributions. Mat. Sb. (N.S.) 101(143), 416-454 (1976)

Publisher's Note Springer Nature remains neutral with regard to jurisdictional claims in published maps and institutional affiliations. 